\title{
Beneficial Effects of Paeoniflorin Enriched Extract on Blood Pressure Variability and Target Organ Damage in Spontaneously Hypertensive Rats
}

\author{
Bo Li, ${ }^{1,2}$ Zheng-Biao Yang, ${ }^{1,3}$ Shan-Shan Lei, ${ }^{1,2}$ Jie Su, ${ }^{1}$ Min-Xia Pang, \\ Chao Yin, ${ }^{4}$ Guo-Yang Chen, ${ }^{4}$ Chao-Wen Shan, ${ }^{1}$ Bo Chen, ${ }^{1}$ Hui-Ming Hu, \\ Su-Hong Chen, ${ }^{1,2,4}$ and Gui-Yuan Lv $^{1}$ \\ ${ }^{1}$ Zhejiang Chinese Medical University, Hangzhou, Zhejiang 310053, China \\ ${ }^{2}$ Zhejiang University of Technology, Hangzhou, Zhejiang 310014, China \\ ${ }^{3}$ Zhejiang Academy of Medical Sciences, Hangzhou 310053, China \\ ${ }^{4}$ Wenzhou Medical University, Wenzhou, Zhejiang 325035, China \\ Correspondence should be addressed to Su-Hong Chen; chensuhong@zjut.edu.cn and Gui-Yuan Lv; zjtcmlgy@163.com
}

Received 30 October 2016; Accepted 20 December 2016; Published 24 January 2017

Academic Editor: Waris Qidwai

Copyright (c) $2017 \mathrm{Bo} \mathrm{Li}$ et al. This is an open access article distributed under the Creative Commons Attribution License, which permits unrestricted use, distribution, and reproduction in any medium, provided the original work is properly cited.

Blood pressure variability (BPV) is associated with the development and progression of severe target organ damage (TOD). This study aims to evaluate the protective effect of paeoniflorin enriched extract from Radix Paeoniae Alba (PG) on BPV and TOD in spontaneously hypertensive rats (SHR). All SHR were orally treated with distilled water, metoprolol (MP, 20 mg/kg), and PG (PG$\mathrm{H}, 90 \mathrm{mg} / \mathrm{kg}$ or PG-L, $30 \mathrm{mg} / \mathrm{kg}$ ) for a single time or daily for 7 weeks. The 24-hour dynamic blood pressure was monitored and then calculated BPV including long- and short-term systolic blood pressure variability (SBPV), diastolic blood pressure variability (DBPV), mean blood pressure variability (MBPV), and heart rate variability (HRV) as well as the 24-hour-SBP, 24-hour-DBP, and 24-hour-MBP. The protective effects of PG on TOD were observed by histopathologic and biochemical detection. The results indicated that long- and short-term SBPV, DBPV, MBPV, and HRV as well as 24-hour-SBP, 24-hour-DBP, and 24-hour-MBP showed no significant changes after single-dose administration of PG and significantly decreased after administration with PG for 7 weeks. PG could also markedly improve the damage of aorta, heart, kidney, and brain. This study suggested that PG could notably reduce BPV, stabilize blood pressure, and mitigate TOD in SHR.

\section{Introduction}

Hypertension is one of the most popular risk factors affecting cardiovascular disease. Blood pressure (BP) sustaining high systolic pressure $\geq 140 \mathrm{mmHg}$ or diastolic pressure $\geq 90 \mathrm{mmHg}$ may be defined as hypertension [1]. According to epidemiology research, the occurrence of hypertension is persistent and shows an increasing trend. The global population suffering from hypertension is predicted to reach 1.56 billion by 2025 [2]. About $25.2 \%$ of people over 18 years old were diagnosed with hypertension in 2012 in China and almost $35.5 \%$ in Beijing [3]. The problem is shown not only by the large number of illness, but also by severe incidental symptoms of target organ damage (TOD). Fortunately, studies have been conducted on blood pressure variability (BPV) as an important risk factor inducing TOD $[4,5]$.

$\mathrm{BPV}$ is associated with the development and progression of severe target organ lesion of the brain, heart, kidney, and vessels, together with increased risk of cardiovascular disease and increased mortality. Thus, the treatment of hypertension should aim not only at decreasing BP but also at reducing BPV [6]. In recent decades, drug treatment of hypertension has focused on controlling BPV. Visit-to-visit research had found that antihypertensive therapeutic drugs of calcium channel blockers (CCB)/diuretics could reduce variability in systolic blood pressure (SBP) and angiotensin-receptor blocker (ARB)/CCB could reduce the long-term variability [7]. 


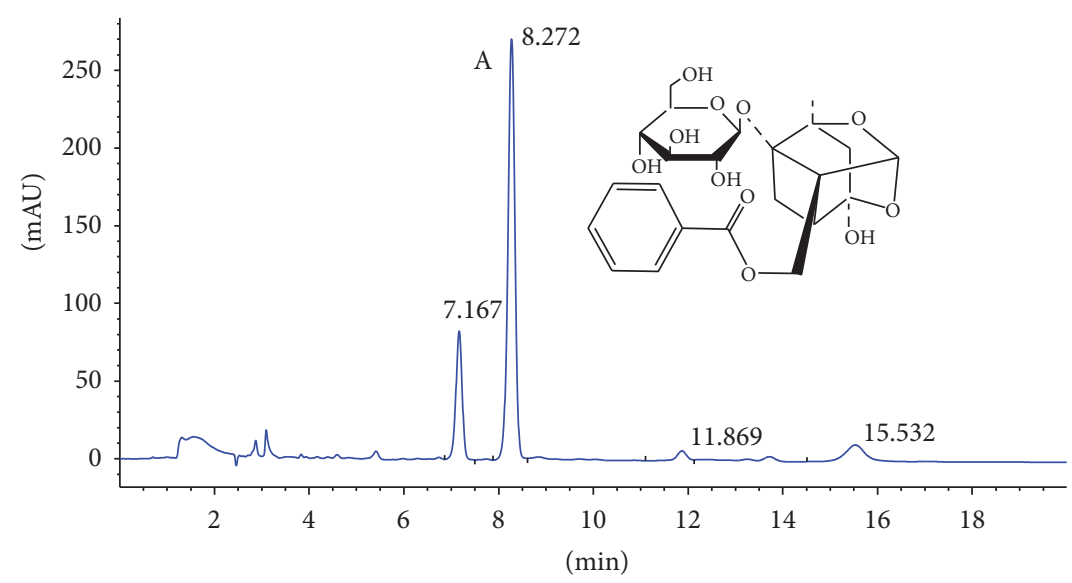

(a)

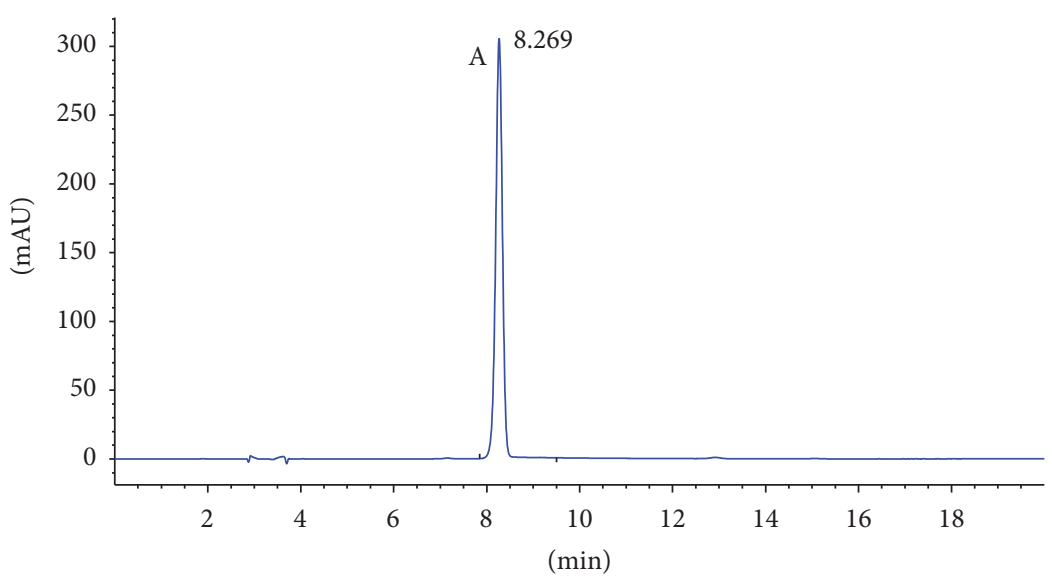

(b)

FIGURE 1: HPLC-DAD analysis of paeoniflorin. (a) HPLC chromatogram of paeoniflorin enriched extract (PG). (b) HPLC chromatogram of standard substance of paeoniflorin. "Peak A" was identified to be paeoniflorin.

Radix Paeoniae Alba (RPA), with paeoniflorin as principal bioactive component, is a traditional Chinese medicine that lowers blood pressure and exhibits anti-inflammatory and antioxidative properties, among others [8]. An increasing number of studies found that paeoniflorin or extract of RPA exerts a positive effect on cardiovascular function [9-11]. The literature reported that the methanol extract of RPA and paeoniflorin could activate nitric oxide synthase (NOS) pathway, thereby increasing nitric oxide (NO) and NOS levels, as well as relaxing blood vessels in vitro [12]. Paeoniflorin and glucosides in RPA can reduce the pressure myocardial vascular remodeling and even myocardial remodeling [13]. In addition, paeoniflorin could reverse hypotensive Wistar rats induced by guanethidine, revealing a two-way method of regulating the effects of $\mathrm{BP}[14]$.

In our previous study, we evaluated the potential of paeoniflorin enriched extract from Radix Paeoniae Alba (PG) as an antihypertensive agent in spontaneous hypertensive rats (SHR) $[15,16]$. We found that PG could decrease blood pressure (SBP, DBP, and MBP) in hypertensive rats, and the mechanism of action was close to its liver protection activity and improvement of endothelial function by reducing ET-1 and increasing NO concentrations. Our survey of literature confirmed that no studies of PG had been conducted to explore BPV and target organ damage in SHR. In our study, we further explored the effect of PG on long- and shortterm variability by administering $P G$ at one time or over a long duration to verify the positive effect on BPV. As BPV could cause severe TOD, histopathologic observation of brain, kidney, heart, and thoracic aorta was also performed.

\section{Animal and Materials}

2.1. Animal. Male spontaneously hypertensive rats (SHR) (forty-eight weeks old) were all purchased from Beijing Vital River Laboratory Animal Technology Co., Ltd., and the licenses numbers of experimental animal were SCXK (Jing) 2012-0001. All procedures were performed according to protocols following the guidelines for the Use and Care of Laboratory Animals published by the Zhejiang province (2009).

2.2. Materials and Reagents. Paeoniflorin enriched extract from Radix Paeoniae Alba (PG) was obtained from Zelang Medical Technology Co. (Nanjing, Jiangsu, China) and the purity was $53 \%$ detected by HPLC analysis (Figure 1). 


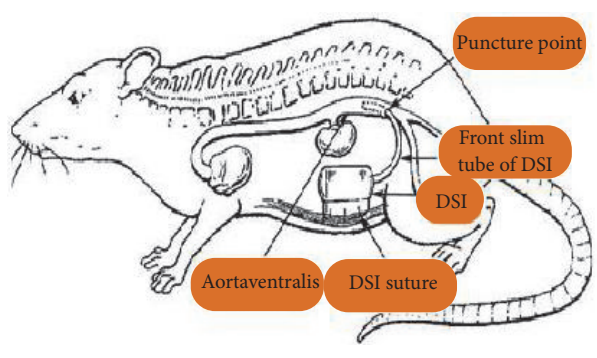

(a)

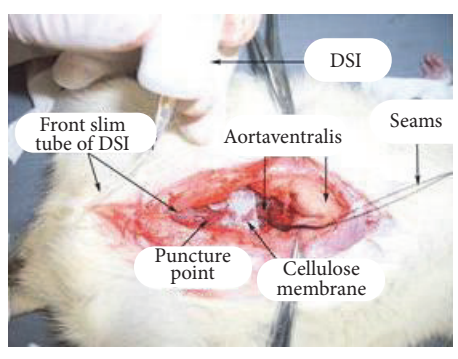

(b)

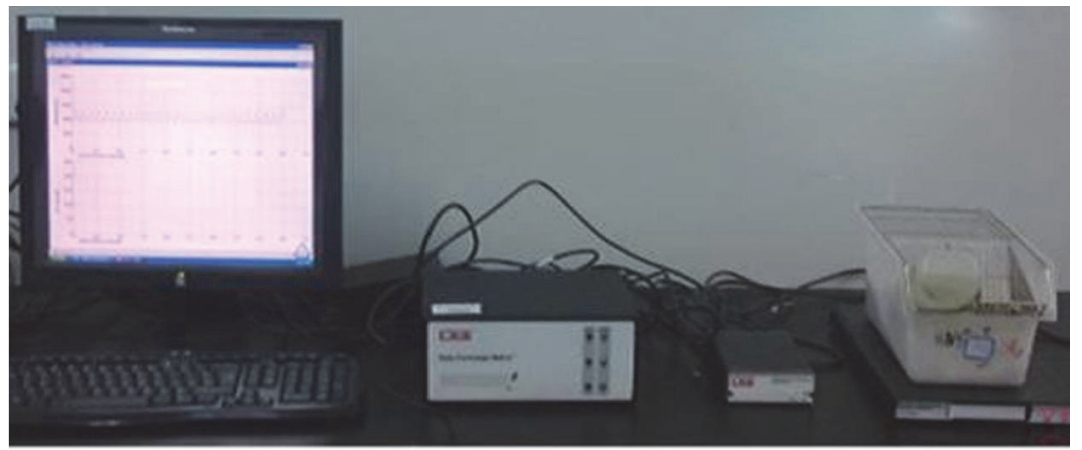

(c)

FIGURE 2: Implantable telemetry technology to monitor dynamic blood pressure in SHR. (a) and (b) The sketch map of intraperitoneal implant site. (c) The transmitting signal device.

Metoprolol Tartrate Tablets were purchased from AstraZeneca Pharmaceutical Co., Ltd. (Lot number 1304136). Urea nitrogen (BUN, Lot number 20140617), creatine (Cr, Lot number 20140627), and uric acid (UA, Lot number 20140523) were obtained from Mei Kang Chemical Co. (Ningbo, Zhejiang, China). Hematoxylin (Lot number 20140919), eosin (Lot number 20140919), and Masson's trichrome kit (Lot number 20140919) were purchased from Nanjing Technology Co., Ltd. (Jiangsu, China). Antibodies against endothelial nitric oxide synthase (eNOS, D9A5L) and cyclooxygenase 2 (COX-2 and D5H5) were from Cell Signaling Technology (Beverly, MA). Mouse and Rabbit Specific HRP/DAB (ABC) Detection IHC kit (ab64264) was from Abcam (Cambridge, USA).

2.3. HPLC Analysis the Purity of Paeoniflorin. Paeoniflorin enriched extract from Radix Paeoniae Alba (PG) was analyzed with HPLC-DAD. The analysis could simply describe that PG sample was diluted with methanol and filtered through a $0.22 \mu \mathrm{m}$ membrane filter before put into the system. The Agilent HPLC1200 (Agilent Technologies Inc., Palo Alto, American) was used to determinate the content of paeoniflorin in extracts by C18 column $(250 \mathrm{~mm}$ $\times 4.5 \mathrm{~mm}$ ). The mobile phases were composed of acetic acid and phosphoric acid solution $(19: 81, V / V)$, and solvent flow rate was $1 \mathrm{ml} / \mathrm{min}$ at column temperature of $25^{\circ} \mathrm{C}$. The injection volume was $5 \mu \mathrm{l}$. The photodiode array detector was set at $230 \mathrm{~nm}$ with a total runtime of $20 \mathrm{~min}$. The HPLC chromatogram of extracts was showed in Figure 1(a).

2.4. Implantable Telemetry Technology to Monitor BPV in $S H R$. Postoperative recovery for one week, selecting three successful surgical SHR, randomly was used for the experiment, based on $24 \mathrm{~h}$ dynamic blood pressure (data were not shown). Rats were sequentially given water (model group, MG), metoprolol (20 mg/kg, MP), and PG (90 mg/kg, PG-H; $30 \mathrm{mg} / \mathrm{kg}, \mathrm{PG}-\mathrm{L}$ ) every 24 hours according to body weight.

After administration, implantable telemetry technology (Data Sciences International, DSI) collected 24-hour blood pressure (BP) immediately, including systolic blood pressure (SBP), diastolic blood pressure (DBP), and mean arterial blood pressure (MBP). Long-term BPV (SBPV, MBPV, and DBPV) was defined as dividing 24-hour BP into 48 sections, each section for $30 \mathrm{~min}$, and calculated the standard deviation of 48 average values, and the short-term BPV (SBPV, MBPV, and DBPV) was defined as the average values of the 48 sections. The long- and short-term heart variability (HRV) were calculated with the same way the long- and short-term $\mathrm{BPV}$, respectively.

The operation was carried out following the procedures presented in literature $[17,18]$. Firstly rats were anaesthetized with $3 \%$ pentobarbital sodium; then abdominal cavity was exposed and abdominal aorta was separated. The second step was to implant C50-PXT device in the intraperitoneal location. The last step was to suture wound and connect the signal to data quest software in computer to monitor BP for 24 hours. During the surgery and recovery, rats were injected intraperitoneally every day to prevent infection with $0.5 \mathrm{ml}$ penicillin (160 thousand $\mathrm{U} / \mathrm{ml}$ ) and rats could take food and water freely. An illustration of intraperitoneal implant site and transmitting signal device was presented in Figure 2.

2.5. Conscious and Freely Moving Animals Dynamic Blood Pressure Analysis System to Monitor BPV in SHR. SHR were 


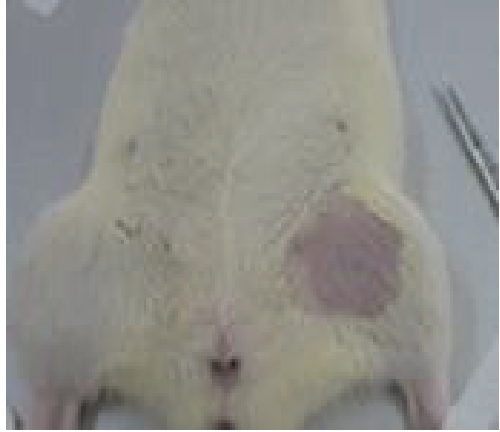

(a)

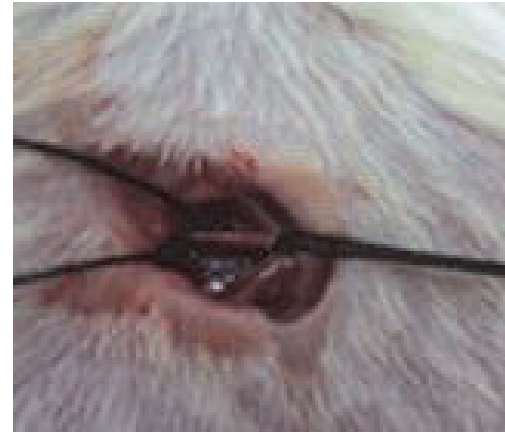

(b)

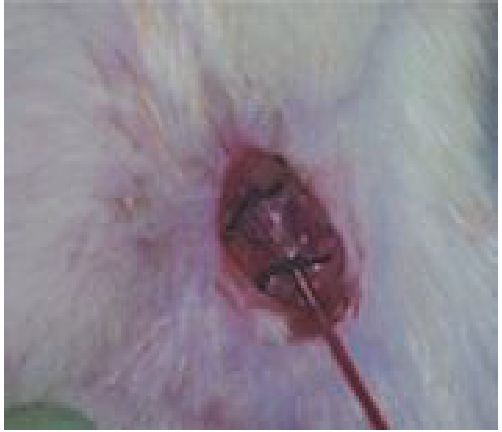

(c)

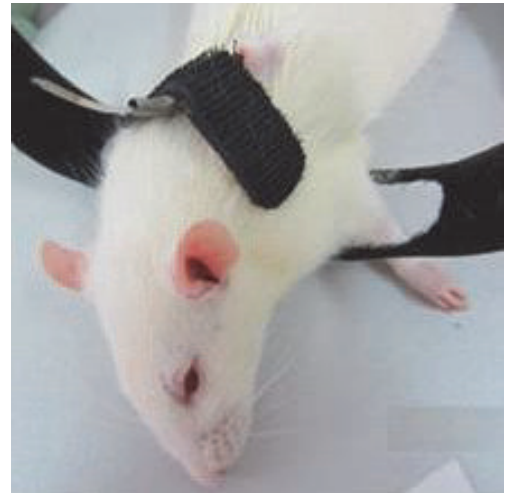

(d)

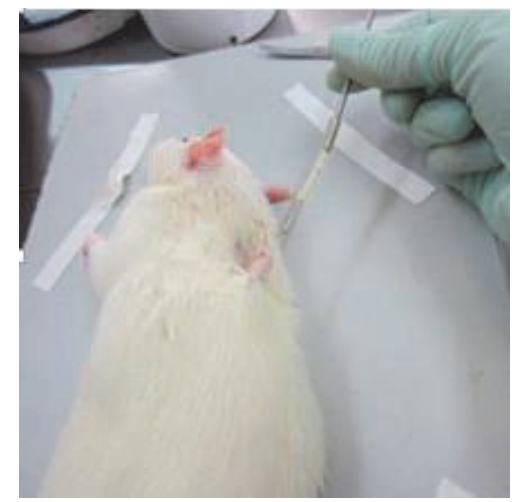

(e)

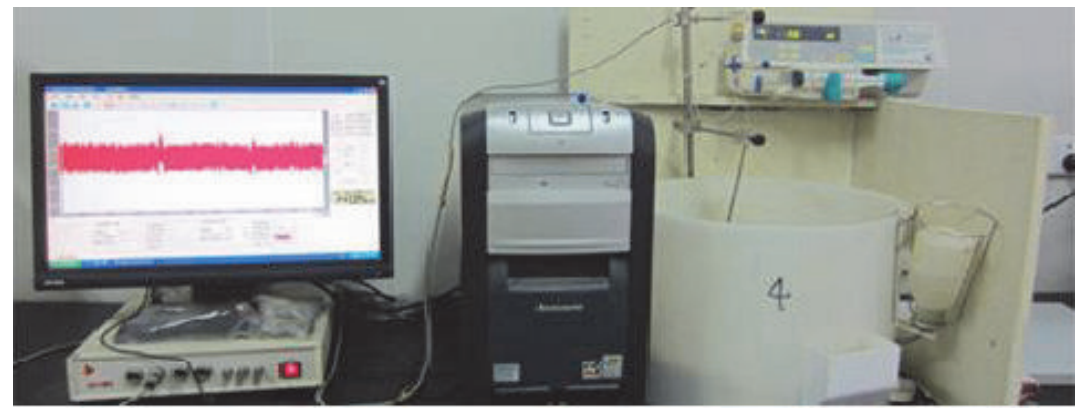

(f)

FIGURE 3: Conscious and freely moving animals dynamic blood pressure analysis system to monitor dynamic blood pressure in SHR. (a)-(e) The sketch map of surgical procedure. (f) The transmitting signal device.

randomly assigned to four groups of eight rats each following as model group (MG), metoprolol positive group $(20 \mathrm{mg} / \mathrm{kg}$, MP), PG high dose group (90 mg/kg, PG-H), and PG low dose group (30 mg/kg, PG-L), based on SBP (data were not shown). Model group was given water, while others were given corresponding drugs according to body weight daily for seven weeks.

With postoperative recovery for 24 hours, level-headed sober animals dynamic blood pressure analysis system (Shanghai Alcott Biotech Co. Ltd., China) recorded 24-hour BP, including SBP, DBP, and MBP. The computing method of long- and short-term SBPV, MBPV, DBPV, and HRV was the same to former part.

The operation was carried out following the procedure presented in literature [19]. Firstly, rats were anaesthetized with $3 \%$ pentobarbital sodium; then the femoral artery was separated and cannula was inserted with the length of weight body $\times 1 \%+1 \mathrm{~cm}$, about $5 \mathrm{~cm}$. Secondly, subcutaneous needle would fix the catheter. At last, level-headed sober animals dynamic blood pressure analysis system was connected and used to monitor BP for 24 hours. During the surgery and recovery, rats were injected intraperitoneally every day to prevent infection with $0.5 \mathrm{ml}$ penicillin (160 thousand $\mathrm{U} / \mathrm{ml}$ ) and rats could take food and water freely. These procedures were displayed in Figure 3.

2.6. Renal Function Detected with Biochemical Indexes. After administration for six weeks, SHR were fasted overnight; then $1.25 \mathrm{ml}$ blood was obtained from ophthalmic venous plexus. Blood were water-bathed for 2 hours at $37^{\circ} \mathrm{C}$ and centrifuged at $3500 \mathrm{rpm}$ for $10 \mathrm{~min}$. The serum was separated to detect 
the biochemical indexes of BUN, Cr, and UA by TBA-40FR automatic clinical chemistry analyzer (Toshiba, Japan).

\subsection{Histopathology and Immunohistochemistry Observation of} Heart, Brain, Kidney, and Aorta. Meanwhile, aorta, heart, kidney, and brain were put into $4 \%$ formalin for fixation. Then, the organs were cut into applicable slice and went through washing and dehydration and embedded to make of tissue wax chunks (MEIKO EC360 Tissue Embedder, Germany). All the specimens were cut into $4 \mu \mathrm{m}$ thickness (LEICARM2245 slicing machine, Germany) and stained by hematoxylin-and-eosin $(\mathrm{H} \& \mathrm{E})$. In addition, the specimens of aorta, heart, and kidney were also used for Masson's trichrome staining. For immunohistochemistry (IHC), Mouse and Rabbit Specific HRP/DAB (ABC) Detection IHC kit was used for the development of reaction of eNOS in aorta, as well as COX-2 in heart and kidney, and tissues were counterstained with hematoxylin. Histopathology observation was used with biological microscope B5-223IEP (Germany).

2.8. Statistical Analysis. All data were expressed as the mean \pm standard deviation and subjected to $t$-test. When compared with the model group before treatment, the data were subjected to paired-sample $t$-tests. When compared with the same period of the model group, the data were subjected to independent-sample $t$-tests. $P$ value of $<0.05$ was considered statistically significant. All analyses were performed by using an updated version of SPSS 15.0 software.

\section{Results}

3.1. Paeoniflorin Enriched Extract Has Slight Effect on 24-Hour Total Blood Pressure of SBP after Single Administration. First, we evaluated the effect of 24-hour dynamic BP in SHR with single dose of metoprolol and paeoniflorin enriched extract (PG). As a result, metoprolol $20 \mathrm{mg} / \mathrm{kg}$ could decrease SBP, $\mathrm{DBP}$, and MBP at the time of $0.5,1$, and $2 \mathrm{~h}$, compared with the model group before treatment $(P<0.05$ and 0.01$)$, and PG $90 \mathrm{mg} / \mathrm{kg}$ exhibited significant influence on SBP at the time of $0.5 \mathrm{~h}(P<0.05)$ (Figures $4(\mathrm{a}) \sim 4(\mathrm{c}))$. In contrast, $\mathrm{PG}$ $30 \mathrm{mg} / \mathrm{kg}$ had no statistic difference on SBP, DBP, and MBP at any time. Meanwhile, the data of 24-hour total SBP, DBP, and MBP showed that PG and metoprolol, administration for just once, had no significantly effect on those parameters (Figures $4(\mathrm{~d}) \sim 4(\mathrm{f}))$.

3.2. Paeoniflorin Enriched Extract Does Not Aggravate Longand Short-Term Blood Pressure Variability of SBPV, DBPV, $M B P V$, and $H R V$ after Single Administration. After single dose, when compared with the model group before treatment, metoprolol $20 \mathrm{mg} / \mathrm{kg}$ could notably increase SBPV, DBPV, and $\operatorname{MBPV}(P<0.05)$, while PG had no visible influence on long- and short-term of SBPV, DBPV, MBPV, and HRV (Figure 5).

3.3. Paeoniflorin Enriched Extract Ameliorates 24-Hour Total Blood Pressure of SBP, DBP, and MBP after Administration for Seven Weeks. From the former part experiment, PG had slight effect on blood pressure for single administration; then the effect of long-time administration performed further. The results of 24-hour dynamic BP suggested that PG and metoprolol all significantly amended the BP at monitoring time, while PG (30 and $90 \mathrm{mg} / \mathrm{kg}$ ) were provided with the high stability of BP obviously (Figures $6(\mathrm{a}) \sim 6(\mathrm{c})$ ). The data of 24-hour total SBP, DBP, and MBP showed that PG $90 \mathrm{mg} / \mathrm{kg}$ could significantly lower those parameters $(P<0.05,0.01)$, and PG $30 \mathrm{mg} / \mathrm{kg}$ could also remarkably decrease 24-hour total SBP and MBP $(P<0.05)$, compared with the model group (Figures $6(\mathrm{~d}) \sim 6(\mathrm{f})$ ).

3.4. Paeoniflorin Enriched Extract Decreases Long- and ShortTerm Blood Pressure Variability of SBPV, DBPV, and MBPV after Administrating for Seven Weeks. Compared with model group, PG $90 \mathrm{mg} / \mathrm{kg}$ significantly improved the long-term blood pressure variability of SBPV, DBPV, and MBPV $(P<$ $0.01)$, as well as the short-term blood pressure variability of SBPV, DBPV, and MBPV $(P<0.01,0.05)$ (Figures $7(\mathrm{a}) \sim 7(\mathrm{c})$ ). And PG $30 \mathrm{mg} / \mathrm{kg}$ and metoprolol $20 \mathrm{mg} / \mathrm{kg}$ had no visible difference on those parameters (Figure 7).

3.5. Protective Effect on Aorta Pathological Changes after Administration with Paeoniflorin Enriched Extract for Seven Weeks. Masson's trichrome staining was used to estimate overall collagen deposition in the aorta as indicated by the density of blue staining (Figures 8(a) and 8(b)). Collagen deposition significantly increased in the model group, while PG mitigated it in a dose-dependent manner. In addition, we also examined pathological changes of the aorta with $\mathrm{H} \& \mathrm{E}$ staining as manifested by the aorta endothelial shedding, the thickness of the media increase, and vascular smooth muscle cells (VSMC) hypertrophy in the model group (Figure 8(c)). To confirm the aorta endothelial lesions furtherly, then we performed the expression of eNOS in the aorta endothelium with IHC, which revealed that the eNOS expression in the aorta endothelium decreased in the model group (Figure $8(\mathrm{~d})$ ). And PG could reverse those lesions. In common, those data provided favorable evidence that PG could alleviate the hypertension induced histopathology injury of aorta.

3.6. Protective Effect on Heart Pathological Changes after Administration with Paeoniflorin Enriched Extract for Seven Weeks. As shown in the heart H\&E and Masson's trichrome staining, the cardiac muscle cells were wider and the nucleus was larger occasionally accompanied by inflammatory cell infiltration and there were lots of collagen deposition in the model group (Figures 9(a) and 9(b)). To further demonstrate inflammatory lesions, we examined the expression of COX-2 in heart with IHC. The results showed that COX-2 was highly expressed in the model group (Figure 9(c)). In contrast, PG could markedly alleviate those symptoms, which hinted that PG protected SHR against hypertension induced cardiac injury.

3.7. Protective Effect on Kidney Pathological Changes and Function after Administration with Paeoniflorin Enriched Extract. In this part, we defined the impact of PG on kidney 


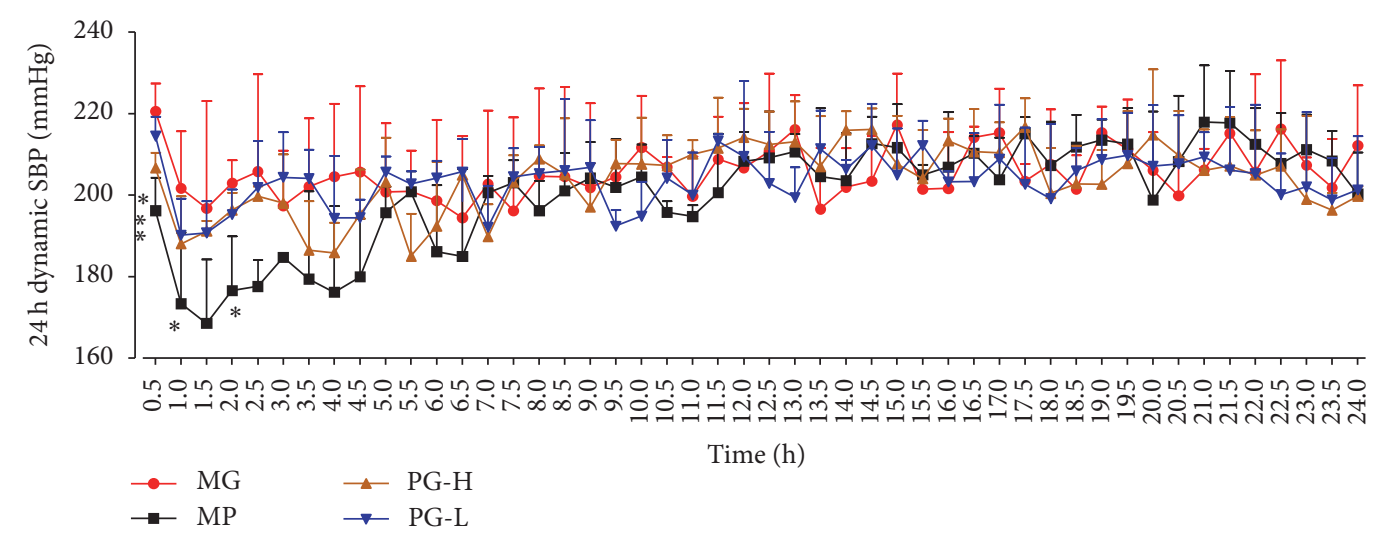

(a)

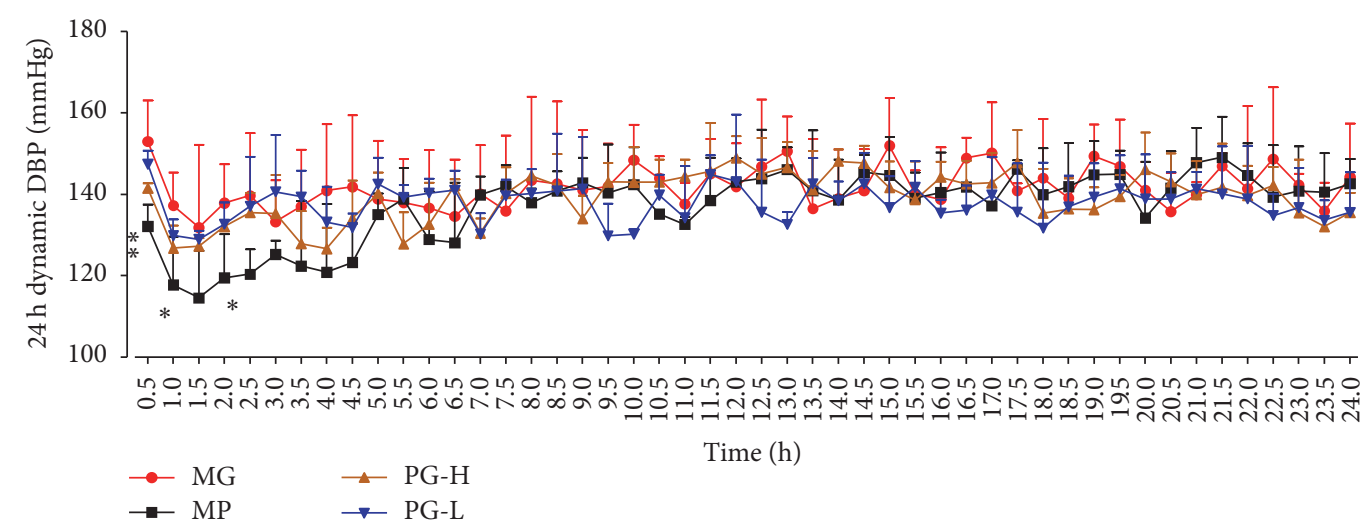

(b)

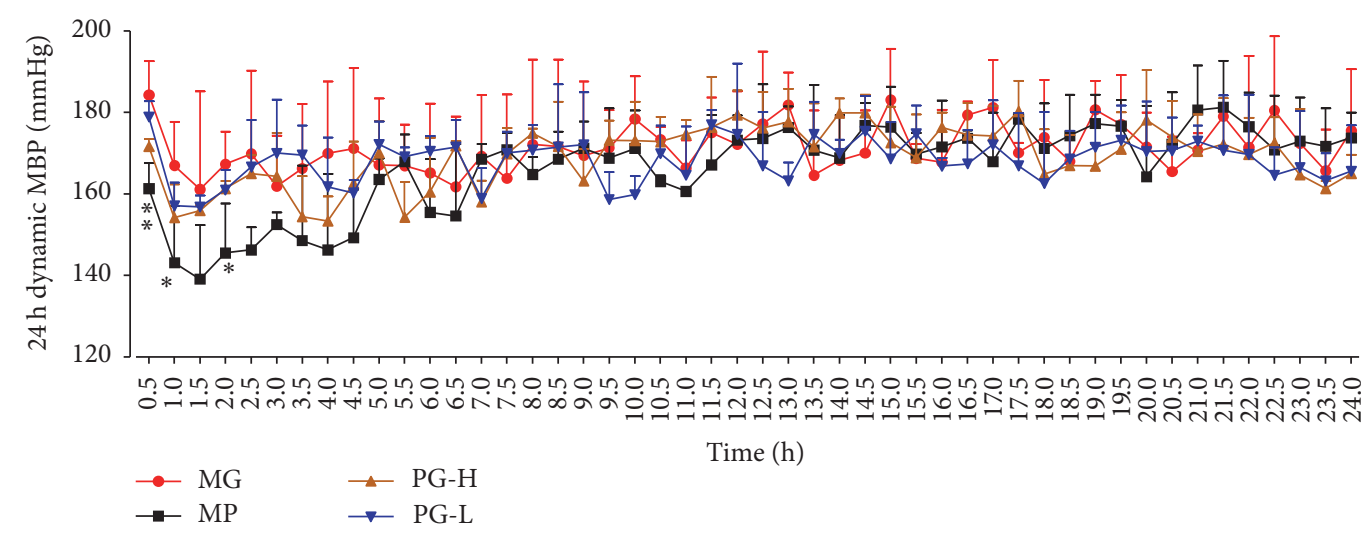

(c)

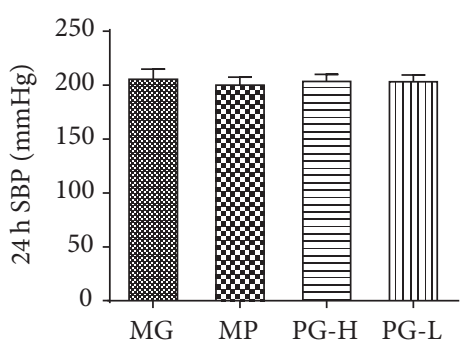

(d)

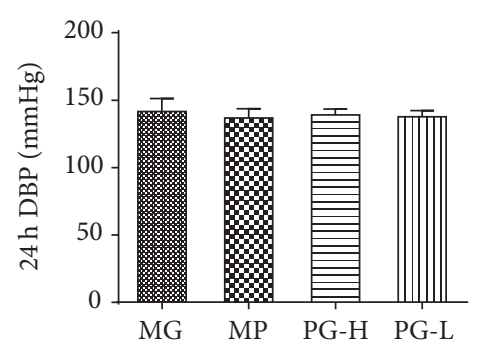

(e)

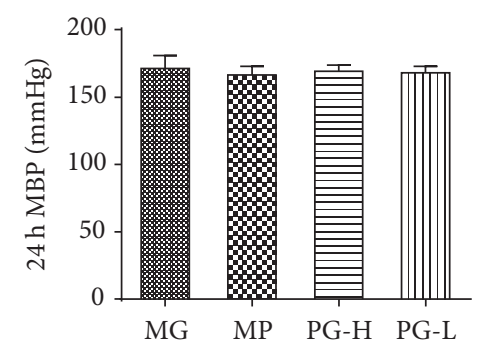

(f)

FIGURE 4: Effect on 24-hour total blood pressure of SBP, DBP, and MBP after being intervened by paeoniflorin enriched extract (PG) at one time. (a) The 24-hour dynamic SBP. (b) The 24-hour dynamic DBP. (c) The 24-hour dynamic MBP. (d) Analysis of 24-hour total SBP. (e) Analysis of 24-hour total DBP. (f) Analysis of 24-hour total MBP. SBP: systolic blood pressure; DBP: diastolic blood pressure; MBP: mean blood pressure; MG: model group before treatment; MP: metoprolol positive group; PG-H: PG high dose group (90 mg/kg); PG-L: PG low dose group $(30 \mathrm{mg} / \mathrm{kg})$. Data were mean $\pm \mathrm{SD}(n=3) .{ }^{*} P<0.05$ versus $\mathrm{MG}$; ${ }^{* *} P<0.01$ versus MG. 


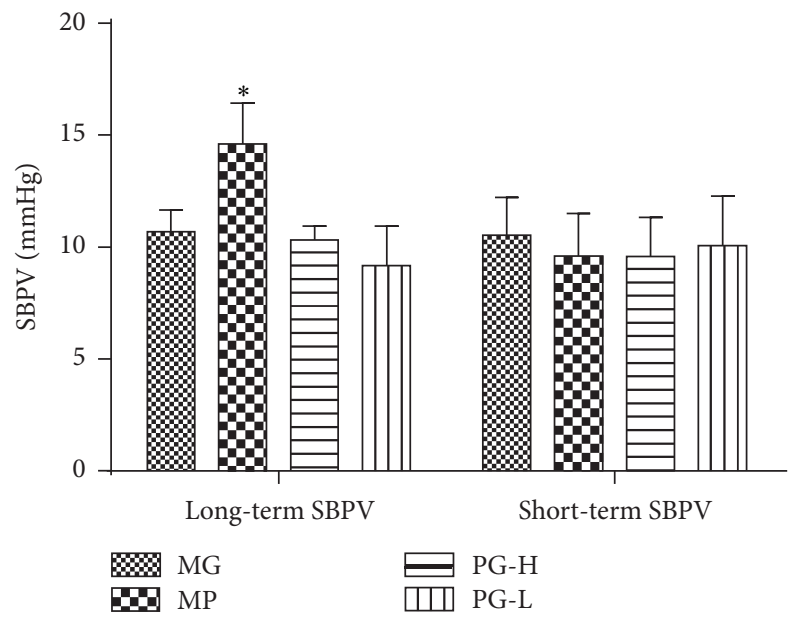

(a)

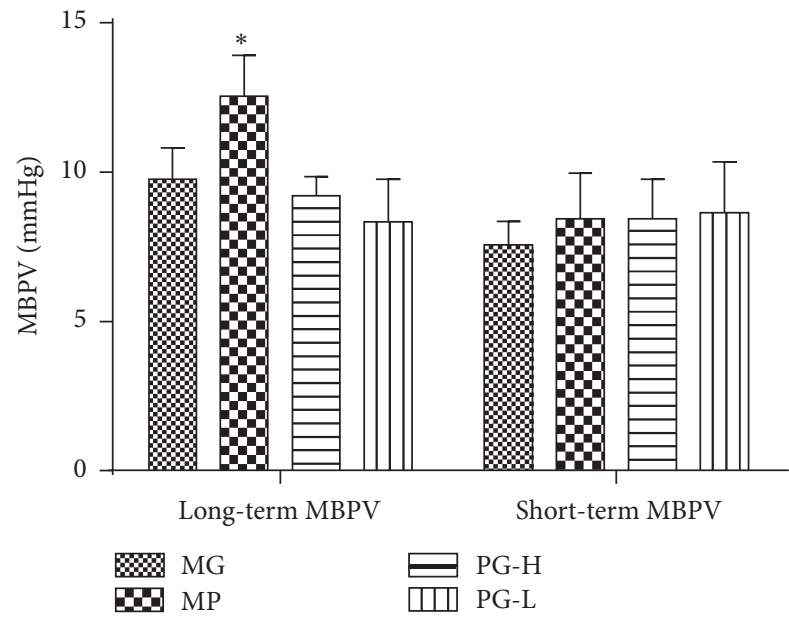

(c)

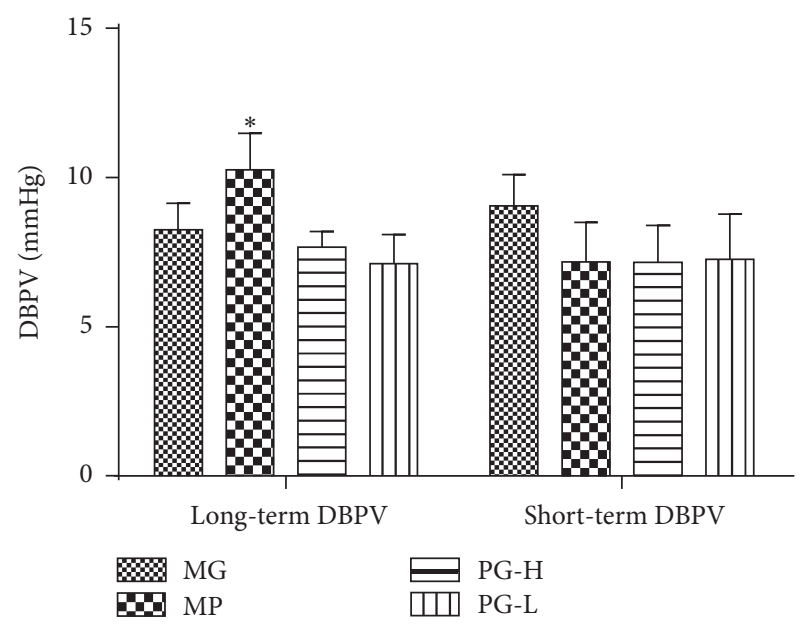

(b)

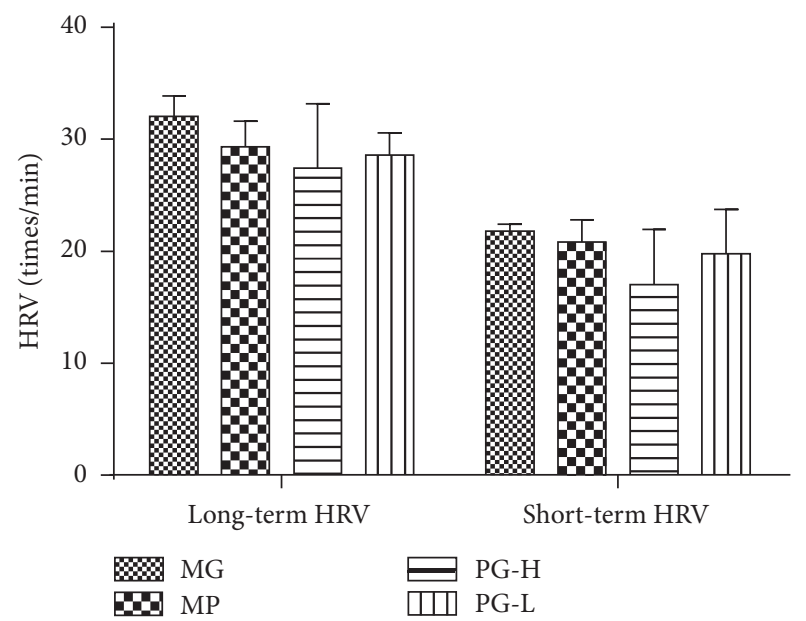

(d)

FIGURE 5: Effect on long- and short-term blood pressure variability of SBPV, DBPV, MBPV, and HRV after being intervened by paeoniflorin enriched extract (PG) at one time. (a) Analysis long- and short-term SBPV. (b) Analysis long- and short-term MBPV. (c) Analysis longand short-term DBPV. (d) Analysis long- and short-term HRV. SBPV: systolic blood pressure variability; DBPV: diastolic blood pressure variability; MBPV: mean blood pressure variability; HRV: heart rate variability; MG: model group before treatment; MP: metoprolol positive group; PG-H: PG high dose group (90 mg/kg); PG-L: PG low dose group (30 mg/kg). Data were mean $\pm \mathrm{SD}(n=3)$. ${ }^{*} \mathrm{P}<0.05 \mathrm{versus} \mathrm{MG}$.

injury by biochemical analysis of the serum BUN, UA, and Cr levels in SHR. Compared with model group, PG $90 \mathrm{mg} / \mathrm{kg}$ and $30 \mathrm{mg} / \mathrm{kg}$ had an significant effect on decreasing the level of UA $(P<0.01)$ (Figure 10(e)). However, PG had no significant effect on serum BUN and Cr. Then, we performed histological analysis with H\&E and Masson's trichrome staining of renal sections. H\&E staining revealed the presence of glomerular wall thickening (Figure 10(a)) and luminal stenosis in the arterioles (Figure 10(b)). Moreover obvious collagen deposition in glomerular wall but no intertubular fibrosis was noted in model group as manifested by the Masson's trichrome staining (Figure 10(c)). And with highly expressed in the model group, the expression of COX-2 in kidney was similar in heart (Figure 10(d)). Of note, PG significantly attenuated these pathological changes. Collectively, our data provided convincing evidence that PG protected SHR against hypertension induced kidney injury.
3.8. Protective Effect on Brain Pathological Changes after Administration with Paeoniflorin Enriched Extract for Seven Weeks. In this section, we examined the protective effect of PG on brain injury with H\&E staining. In the model control group, the cortical cells arranged in disorder and decreased (Figure 11(a)). The cortical vascular endothelial cell was swelling (Figure 11(b)). Meanwhile, by observing the hippocampal CAl area, the pyramidal cell layer became thinner, less, and disordered, and the neurons were degenerated and necrosed obviously (Figure 11(c)). Compared with the model control group, the PG $(90 \mathrm{mg} / \mathrm{kg})$ in different degrees improve the cortical cells lesions, alleviate the swelling of cortical vascular endothelial cells, and attenuate the pyramidal cell layer in hippocampal CA1 area. Although we only used H\&E staining to observe brain lesions, severe brain injury could be clearly observed in the model control group and PG had significant protective effect on the brain injury in SHR. 


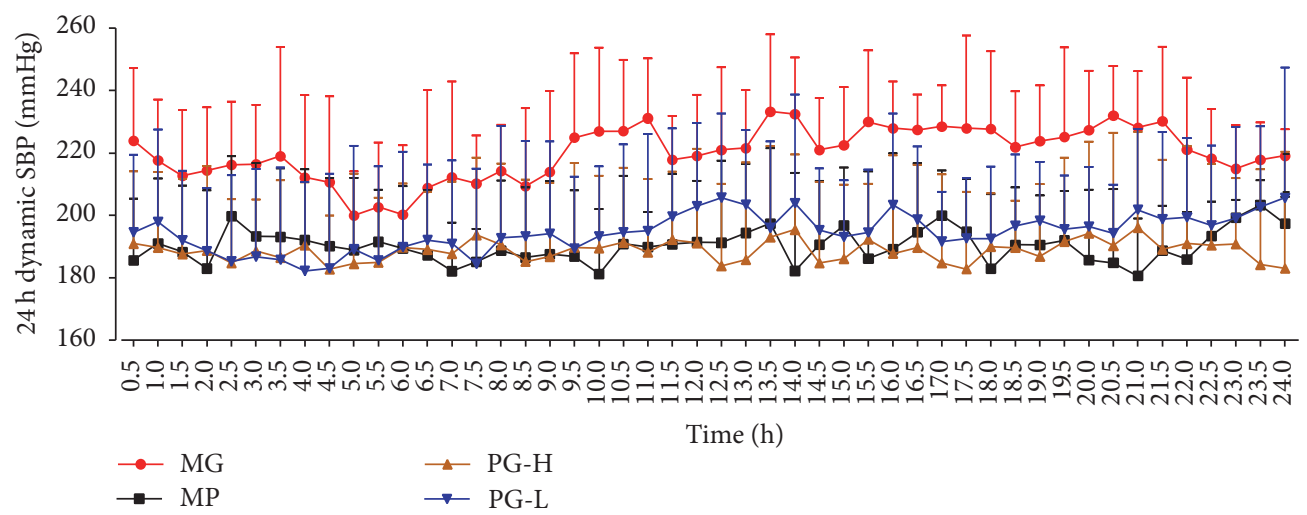

(a)

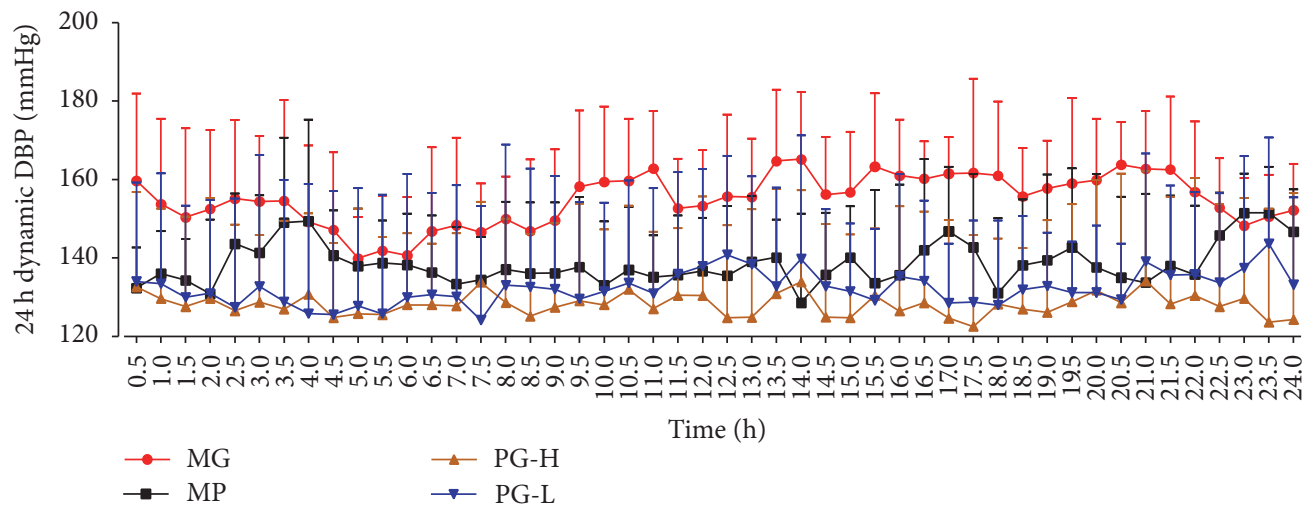

(b)

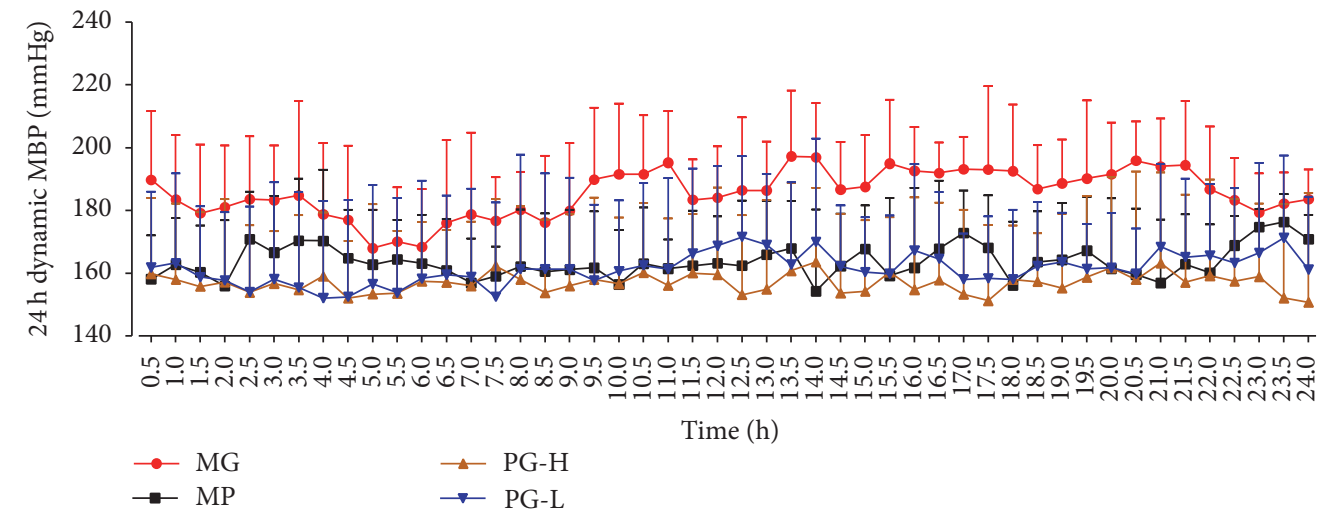

(c)

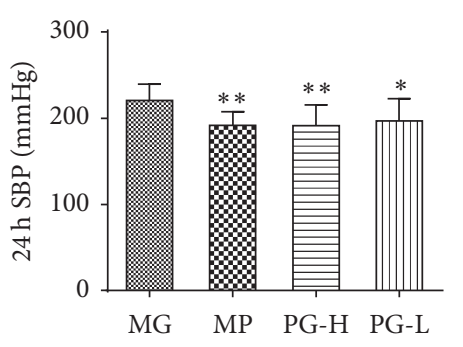

(d)

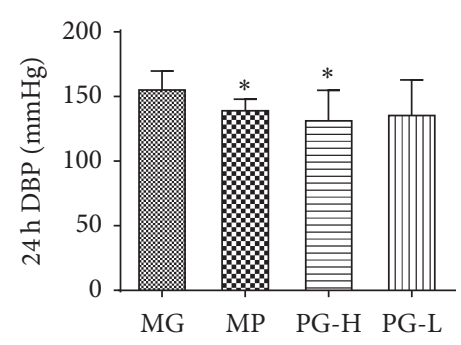

(e)

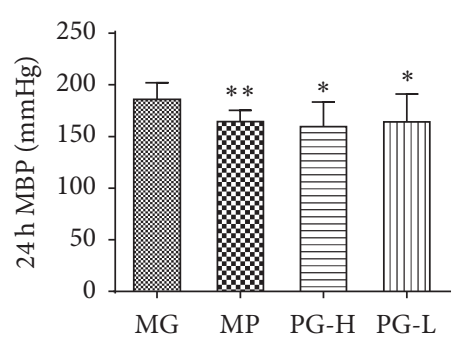

(f)

FIGURE 6: Effect on 24-hour total blood pressure of SBP, DBP, and MBP after being intervened by paeoniflorin enriched extract (PG) for seven weeks. (a) The 24-hour dynamic SBP. (b) The 24-hour dynamic DBP. (c) The 24-hour dynamic MBP. (d) Analysis of 24-hour total SBP. (e) Analysis of 24-hour total DBP. (f) Analysis of 24-hour total MBP. SBP: systolic blood pressure; DBP: diastolic blood pressure; MBP: mean blood pressure; MG: model group; MP: metoprolol positive group; PG-H: PG high dose group (90 mg/kg); PG-L: PG low dose group $(30 \mathrm{mg} / \mathrm{kg})$. Data were mean $\pm \mathrm{SD}(n=8) .{ }^{*} P<0.05$ versus $\mathrm{MG} ;{ }^{* *} P<0.05$ versus MG. 

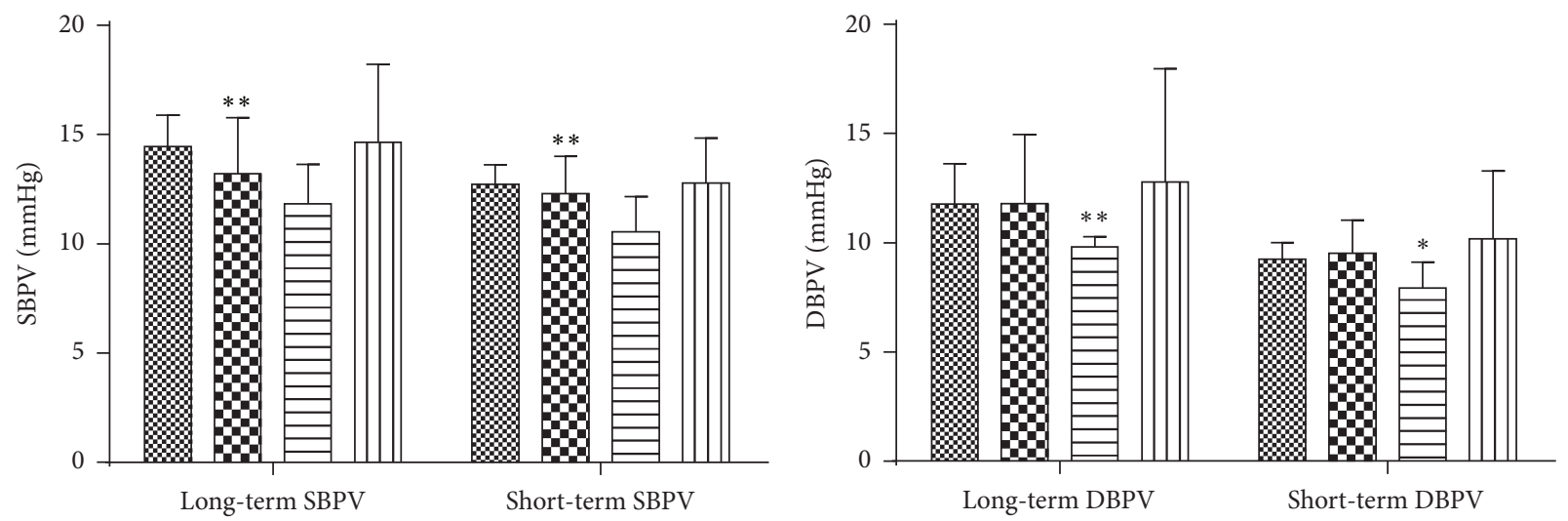

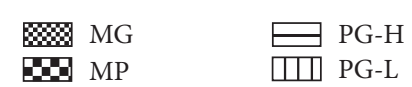

(a)

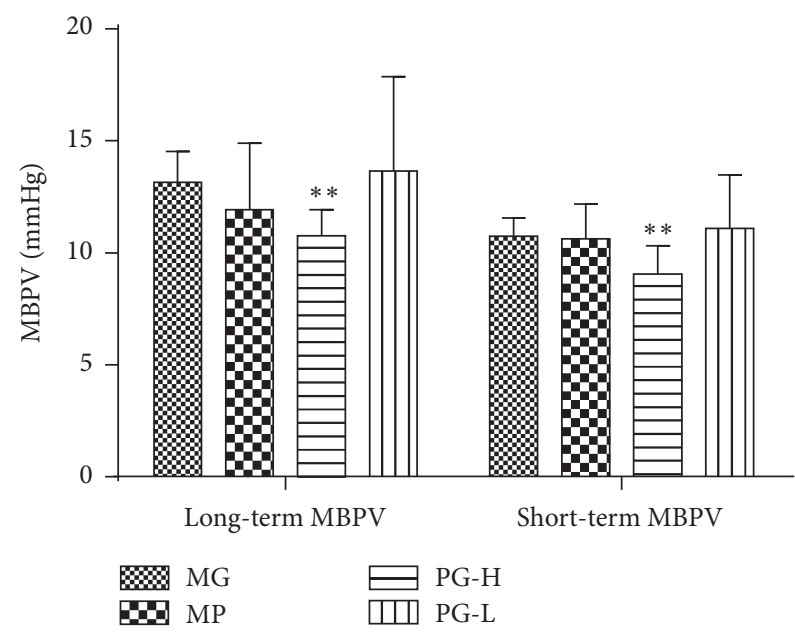

(c)

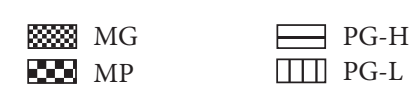

(b)

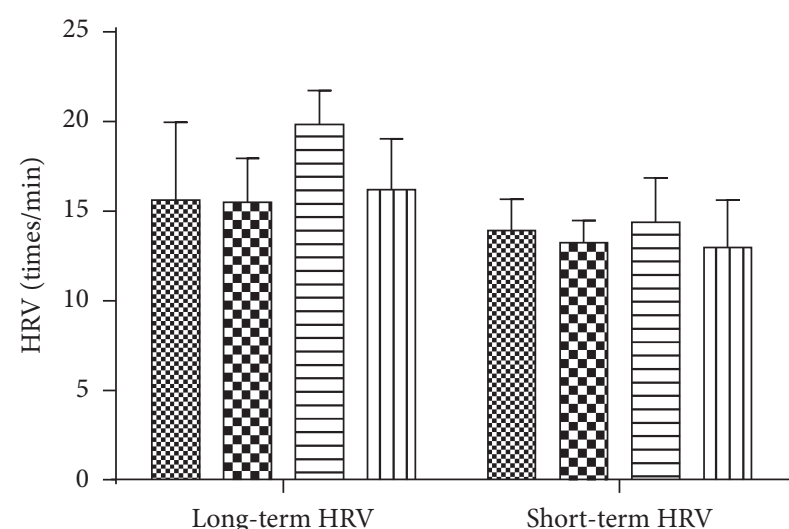

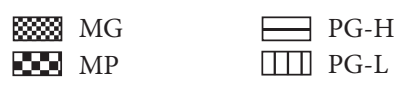

(d)

FIGURE 7: Effect on long- and short-term blood pressure variability of SBPV, DBPV, MBPV, and HRV after being intervened by paeoniflorin enriched extract (PG) for seven weeks. (a) Analysis long- and short-term SBPV. (b) Analysis long- and short-term MBPV. (c) Analysis longand short-term DBPV. (d) Analysis long- and short-term HRV. SBPV: systolic blood pressure variability; DBPV: diastolic blood pressure variability; MBPV: mean blood pressure variability; HRV: heart rate variability; MG: model group; MP: metoprolol positive group; PG-H: PG high dose group (90 mg/kg); PG-L: PG low dose group $(30 \mathrm{mg} / \mathrm{kg})$. Data were mean $\pm \mathrm{SD}(n=8) .{ }^{*} P<0.05$ versus MG; ${ }^{* *} P<0.05$ versus MG.

\section{Discussion}

Hypertension is a disease characterized by high arterial pressure. Sustained high blood pressure leads to cerebral embolism, cardiac failure, renal failure, and other complications. TOD is caused not only by hypertension but also by BPV, which is independent of low mean systolic blood pressure $[4,20]$. Therefore, the treatment of hypertension should focus not only on the effective control of blood pressure, but also on the protection of target organs to reduce complications [6]. Many researchers confirmed that BPV could cause TOD independently, even within normal blood pressure $[21,22]$. There is emanating evidence that BPV is an independent predictor of hypertensive TOD and cardiovascular events [23]. An illustration of TOD is presented in Figure 12.
In the early stage of our research, PG exhibited a definite antihypertensive effect on SHR through liver protection activity and improvement of endothelial function by regulating serum NO and endothelin (ET) levels. However, the effect of PG on blood pressure fluctuation has not been evaluated. On the basis of the previous research, singledose and long-term administration of PG were conducted to investigate its effect on BPV in SHR in this study. The experimental results showed that single-dose administration of PG, unlike metoprolol, significantly reduced blood pressure in rats initially, without aggravating long- and shortterm BPV. Long-term administration of PG could not only significantly reduce the $24 \mathrm{~h}$ blood pressure but also decrease BPV (SBPV, DBPV, and MBPV). By contrast, metoprolol significantly reduced the $24 \mathrm{~h}$ blood pressure; however the trend fluctuated, showing no significant effect on SBPV, 

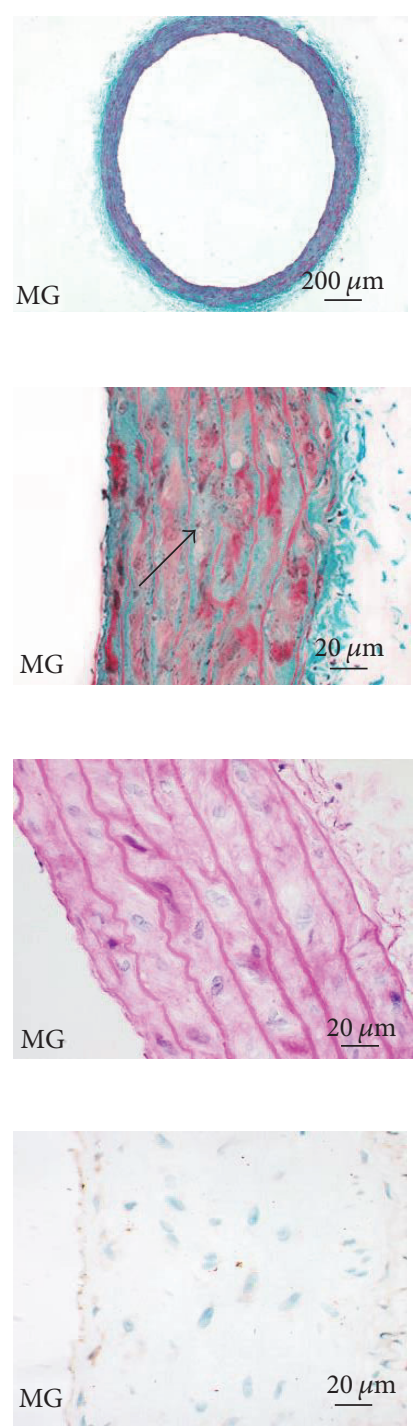

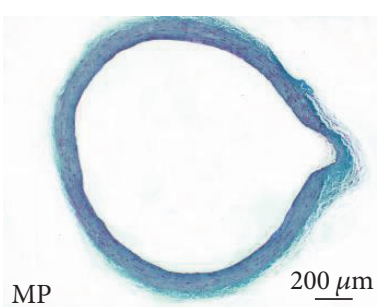

MP
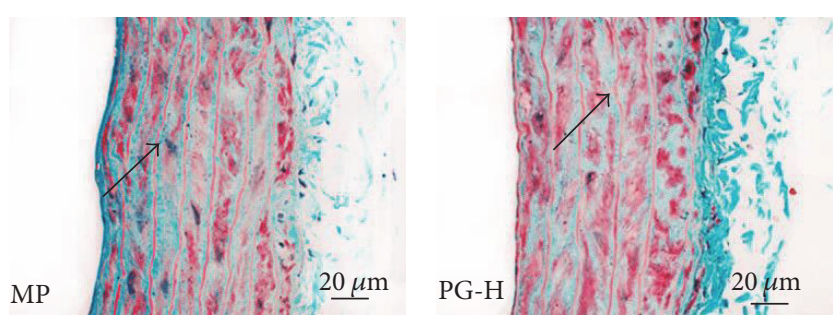

(b)
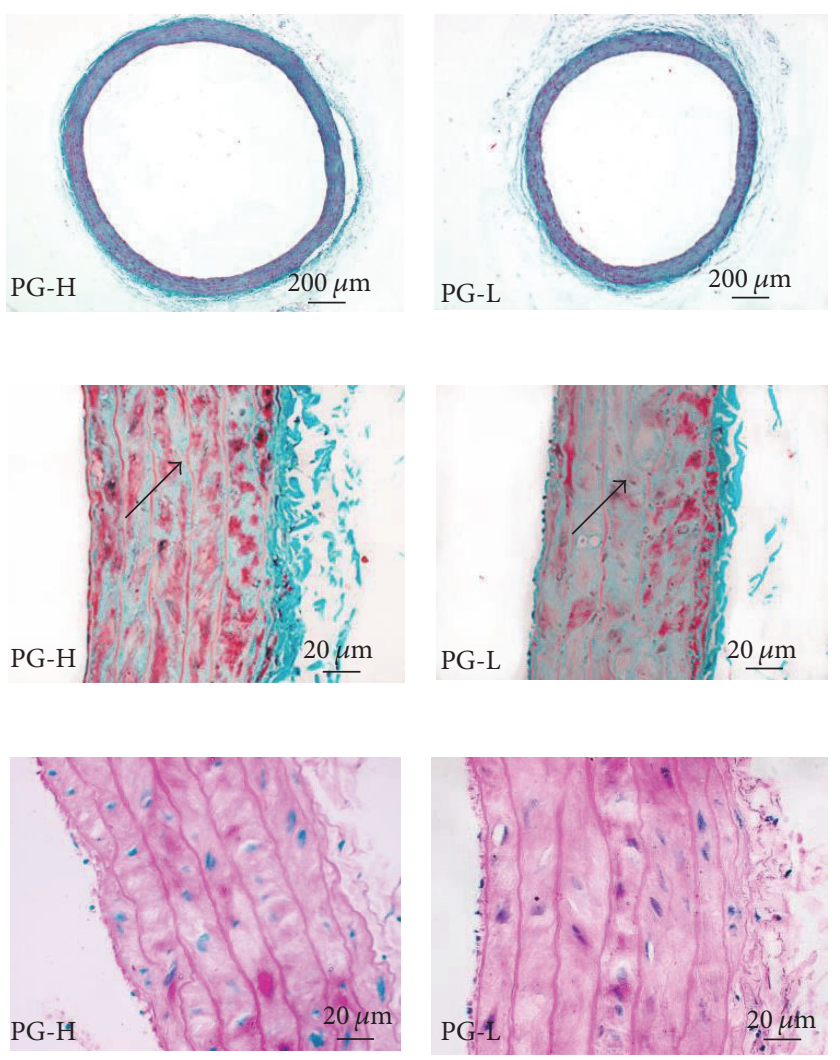

(c)

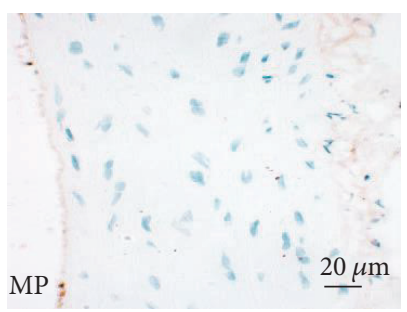

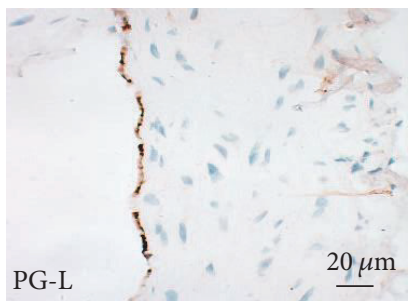

(d)

FIGURE 8: Effect on vascular lesions after being intervened by paeoniflorin enriched extract (PG) for seven weeks. (a) Representative photomicrograph of histopathologic observation for aorta by Masson's trichrome staining ( $\times 40)$. (b) Representative photomicrograph of histopathologic observation for aorta by Masson's trichrome staining $(\times 400)$. (c) Representative photomicrograph of histopathologic observation for aorta by hematoxylin-and-eosin staining $(\mathrm{H} \& \mathrm{E})(\times 400)$. (d) Representative photomicrograph of the eNOS expression in aorta by immunohistochemistry (IHC) $(\times 400)$. MG: model group; MP: metoprolol positive group; PG-H: PG high dose group (90 mg/kg); PG-L: PG low dose group $(30 \mathrm{mg} / \mathrm{kg})$.

DBPV, and MBPV. This effect might be the advantage of using PG as an antihypertensive. The effect of $\beta$-blockers on blood pressure fluctuation remains inconclusive. Betablockers might increase in enhanced BPV [20], which may be attributed to nonselective $\beta$-blockers; high selectivity in $\beta$-blockers exerts no such effect [24].

Vascular cell proliferation, apoptosis, inflammation, fibrosis, and other complex processes change the vascular structure in patients with BPV [25]. The SHR is a stable model for examining the development and complications of hypertension. Increased collagen deposition, endothelial cell abnormalities, and abnormal aortic wall cell proliferation of the media were observed in the 42-week-old SHR [26].
The SHR exhibited a significant reduction of endothelial nitric oxide synthase (eNOS) protein expression in the aortic endothelium [27]. An earlier study suggested that paeoniflorin could promote blood vessel wall function by releasing the relaxation factor of $\mathrm{NO}$ on isolated thoracic aorta rings of SD rats [12]. Likewise, our previous research proved that PG could upregulate serum NO in SHR [15]. Endothelial NOS is the main limiting factor of NO generation, forcefully causes vasodilation, and inhibits the proliferation of vascular smooth muscle cells [28]. The findings in the current study suggested that PG could improve endothelial shedding, relieve hypertrophy of smooth muscle cells, improve collagen fiber hyperplasia, and increase eNOS expression in the 

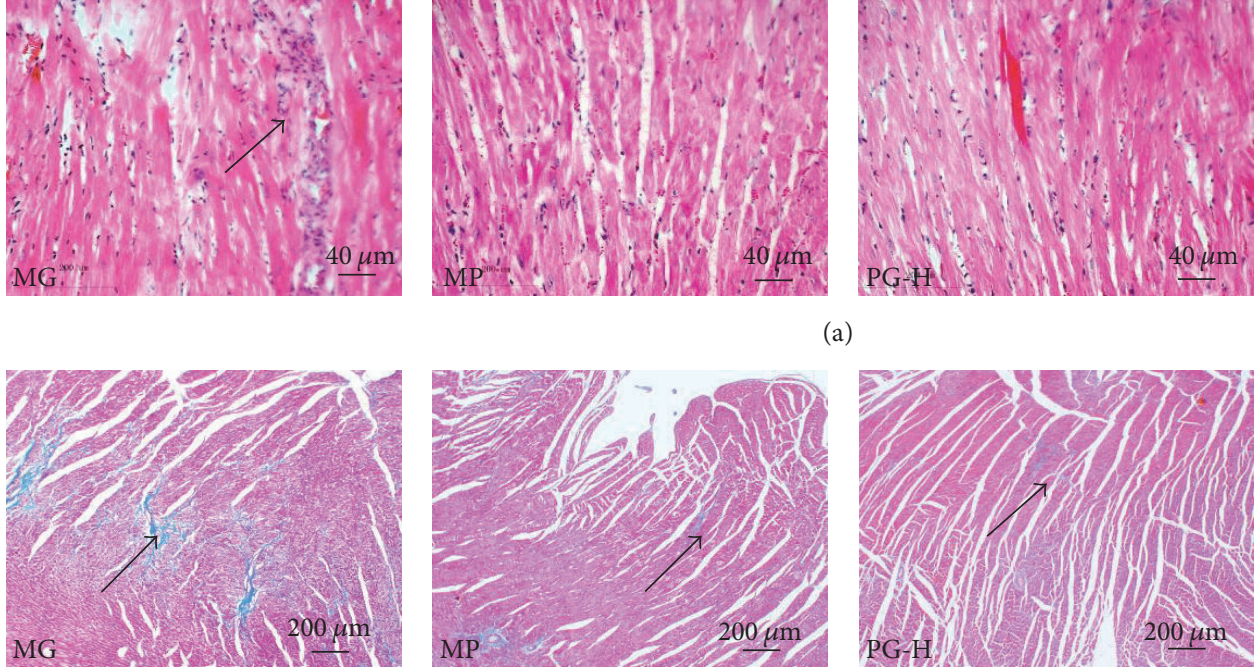

(b)
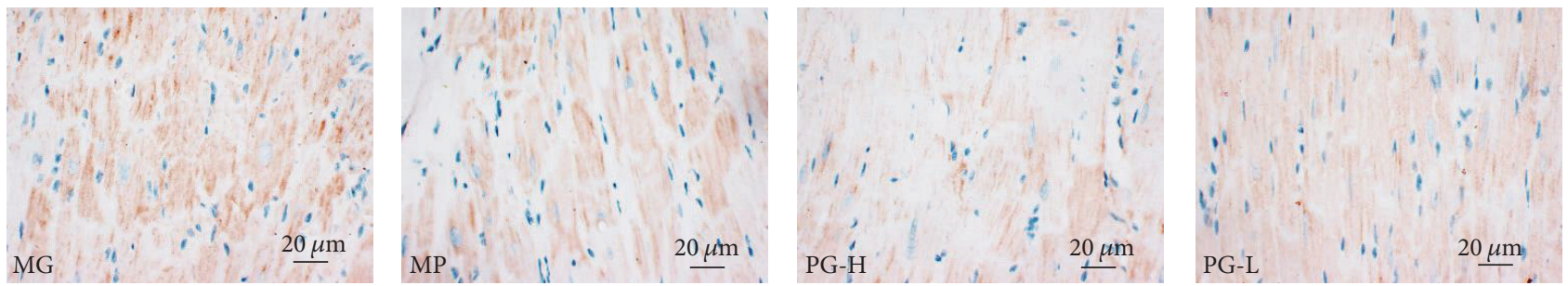

(c)

FIGURE 9: Effect on histopathology of heart after being intervened by paeoniflorin enriched extract (PG) for seven weeks. (a) Representative photomicrograph of histopathologic observation for heart by hematoxylin-and-eosin staining (H\&E) (×200). (b) Representative photomicrograph of histopathologic observation for heart by Masson's trichrome staining $(\times 40)$. (c) Representative photomicrograph of the COX-2 expression in heart by immunohistochemistry $(\mathrm{IHC})(\times 400)$. MG: model group; MP: metoprolol positive group; PG-H: PG high dose group (90 mg/kg); PG-L: PG low dose group (30 mg/kg).

aorta, which hinted that PG could alleviate the hypertension induced histopathology injury of aorta.

Enhanced BPV could trigger a change in pathological cardiac hypertrophy via mechanical stress fluctuation in the cardiomyocytes [29]. That is to say, increased BPV may be responsible for the pathogenesis of hypertrophic cardiac response [30]. The clinical test showed that an increase in BPV over 24-hour evaluation period with ambulatory blood pressure monitoring was linked to a higher degree of hypertensive cardiovascular complications [31,32]. Studies indicated that BPV was highly correlated with cardiovascular complications, and short-term variability could predict the close links to BPV and early left ventricular systolic dysfunction $[33,34]$. BPV also changes in the myocardial structure [35]. SHR aged 56 weeks developed end-stage hypertensive heart disease, enlargement of cardiomyocytes enlargement, and fibrosis [36], which were also were found in SHR aged 20 weeks [37]. Moreover, the expression of COX-2 in cardiomyocytes was significantly correlated with their size [38], and COX-2 might be one of the important indicators of systemic inflammation [39, 40]. Paeoniflorin has been reported to prevent upregulations of proinflammatory mediator COX-2 in ischemia-induced brain damage and rheumatoid arthritis rats [41, 42]. The results obtained in this study revealed that PG could improve myocardial inflammation, ameliorate collagen deposition, and decrease COX-2 expression in heart.

The kidneys target organs that not only are prone to hypertension induced injury but also are also involved in exacerbating the development of hypertension. An increase in short-term BPV may be positively correlated with impaired renal function, as determined by microalbuminuria or glomerular filtration rate $[27,28]$. Glomeruli, tubules-interstitium, and renal vascular lesions were significantly increased in 12-week-old SHR [43, 44]. Renal COX-2 expression was also increased in hypertension mouse [43]. In this study, histopathologic observation of renal tissues indicated that glomerular arteries led to stenosis, thickening of the glomerular wall capsule, collagen deposition, and increase in COX-2 expression in the SHR model. PG could improve the aforementioned symptoms suggesting that PG exhibited a renal protective effect in SHR.

BPV could also lead to brain damage, including cerebral vascular lesions and histomorphological changes in the brain [45]. Cerebrovascular lesions are mainly manifested as hypertrophic and remodeling lesions. Histomorphological changes mainly occur in the frontal lobe, occipital lobe, and hippocampus. Previous studies have indicated that SHR share behavioral and neuropathological characteristics in 

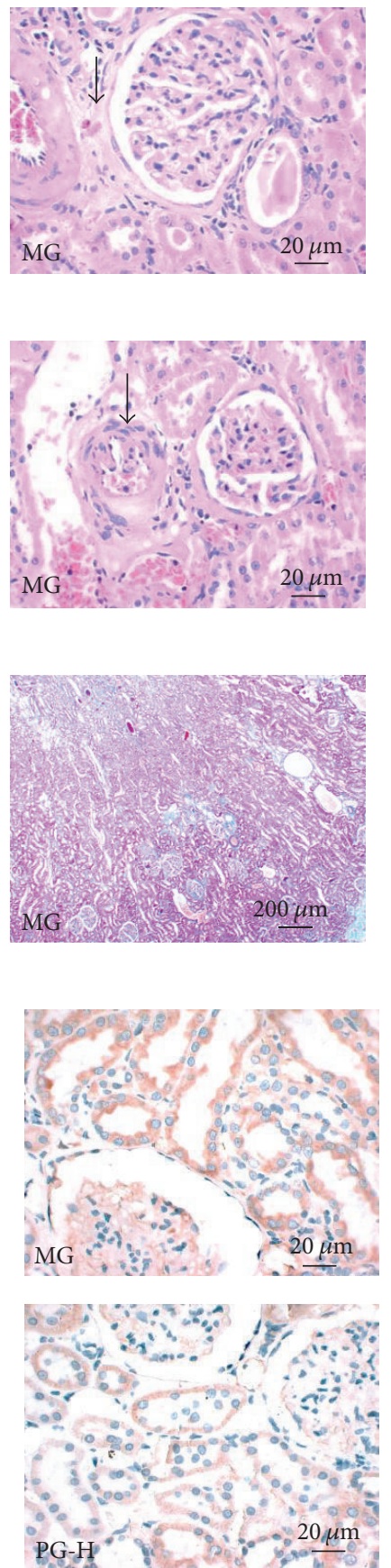

(d)
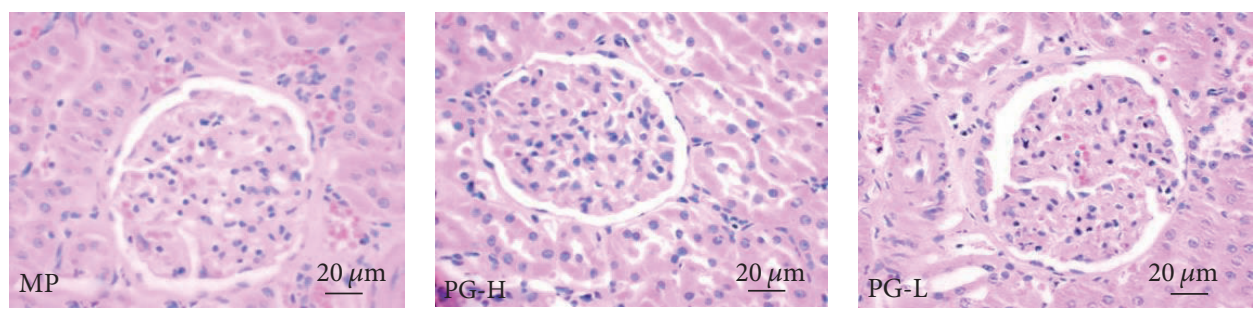

(a)
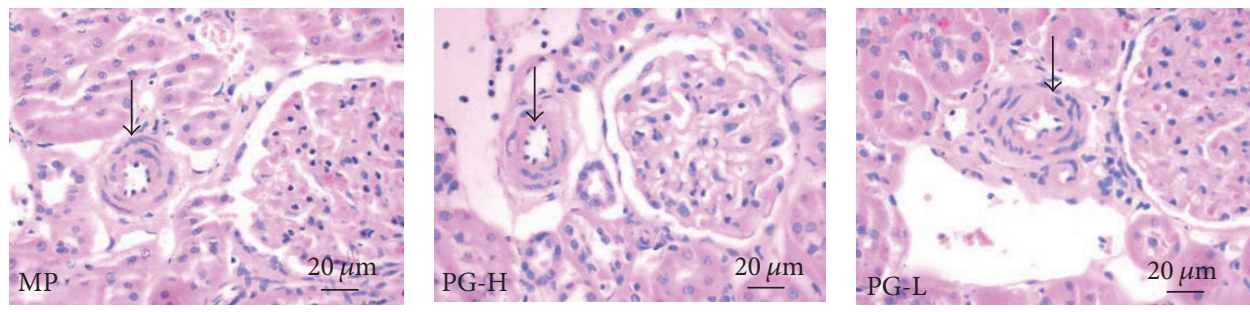

(b)
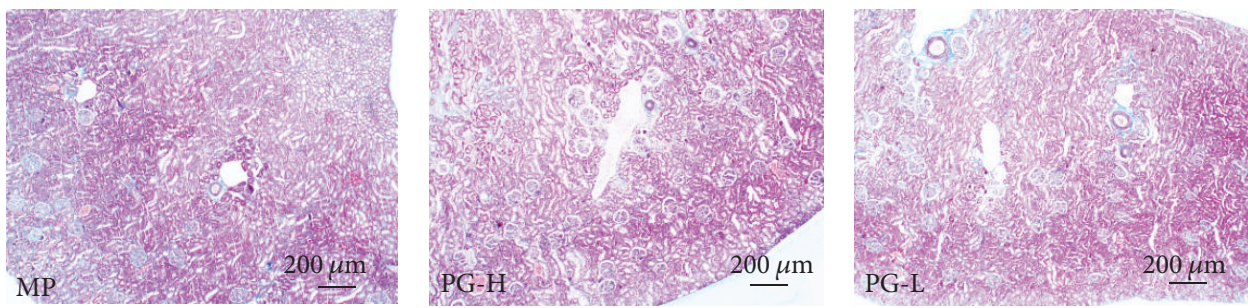

(c)
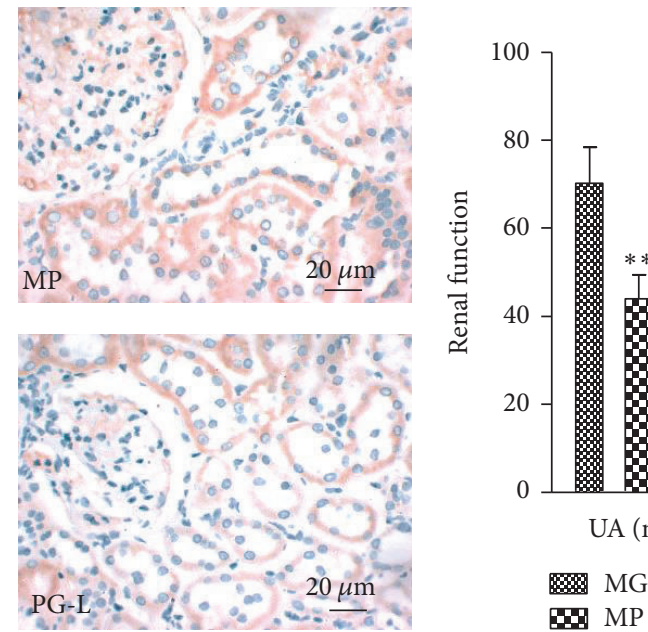

3.1. MP

\section{严 PG-H}

(e)

FIGURE 10: Effect on histopathology of kidney and renal function after being intervened by paeoniflorin enriched extract (PG). (a) Representative photomicrograph of histopathologic observation for glomerulus by hematoxylin-and-eosin staining $(\mathrm{H} \& \mathrm{E})(\times 400)$. (b) Representative photomicrograph of histopathologic observation for glomerular afferent arteries by H\&E $(\times 400)$. (c) Representative photomicrograph of histopathologic observation for kidney by Masson's trichrome staining $(\times 40)$. (d) Representative photomicrograph of the COX-2 expression in kidney by immunohistochemistry (IHC) $(\times 400)$. (e) The renal function indexes of serum UA, BUN, and Cr. MG: model group; MP: metoprolol positive group; PG-H: PG high dose group (90 mg/kg); PG-L: PG low dose group (30 mg/kg). Data were mean $\pm \mathrm{SD}(n=8) .{ }^{* *} P<0.01$ versus MG.

22-week-old SHR [46]. Paeoniflorin could attenuate brain damage in rats and mouse via inflammatory signaling pathways $[47,48]$. In this study, PG could variably increase the number of cortical cells, alleviate the swelling of cortical vascular endothelial cells, and attenuate the pyramidal cell layer in the hippocampal CA1 area.

The current animal models, used to study for hypertension, are mainly hereditary hypertensive animal models 

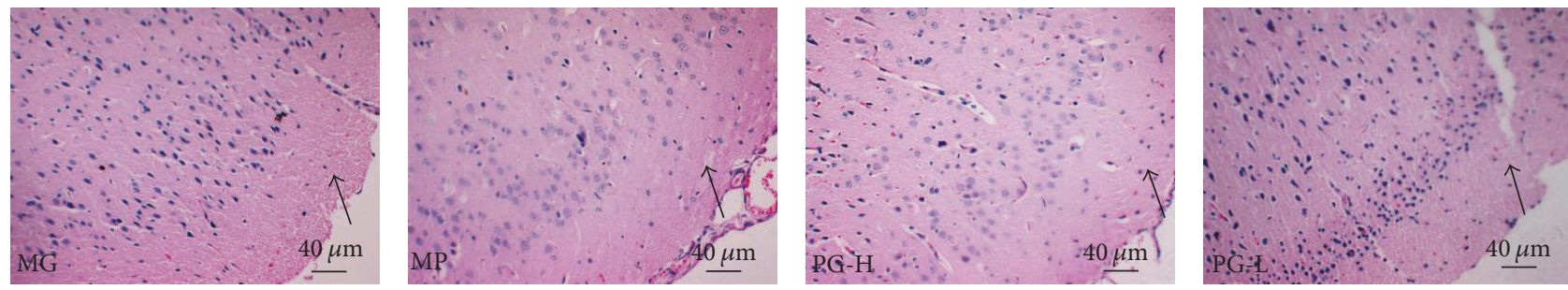

(a)
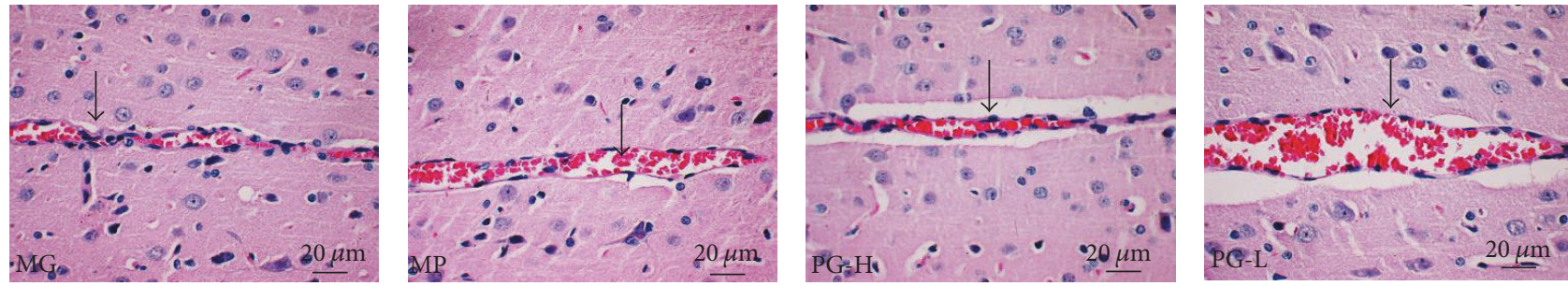

(b)
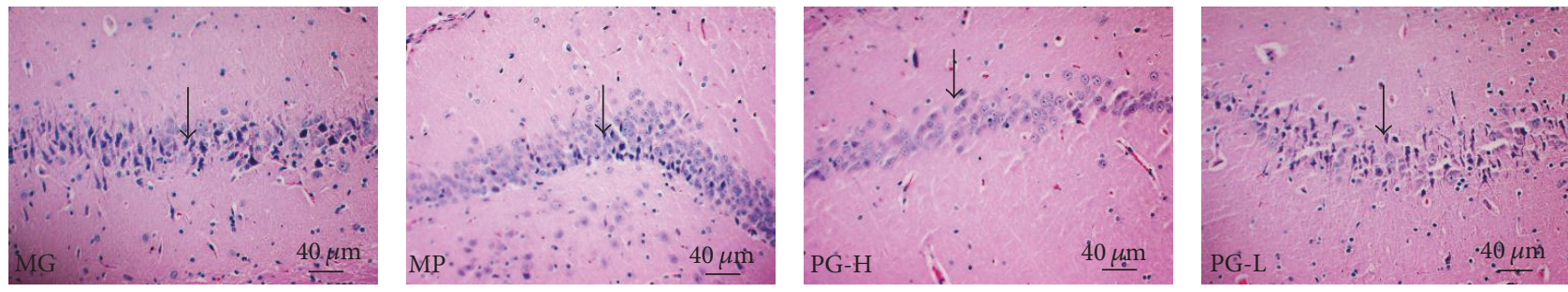

(c)

FIGURE 11: Effect on histopathology of brain after being intervened by paeoniflorin enriched extract (PG) for seven weeks. (a) Representative photomicrograph of histopathologic observation for cerebral cortex by hematoxylin-and-eosin staining $(H \& E)(\times 200)$. (b) Representative photomicrograph of histopathologic observation for cerebral cortex blood vessel by H\&E $(\times 400)$. (c) Representative photomicrograph of histopathologic observation for hippocampal CA1 area by H\&E $(\times 200)$. MG: model group; MP: metoprolol positive group; PG-H: PG high dose group (90 mg/kg); PG-L: PG low dose group (30 mg/kg).

(spontaneously hypertensive rats (SHR) $[49,50]$, strokeprone spontaneously hypertensive rats [51], Dahl saltsensitive hypertensive rats [52], etc.), renovascular hypertensive animal models (2-kidney-1-clip, 2K1C [53] and 2-kidney2-clip, $2 \mathrm{~K} 2 \mathrm{C}$ [54], etc.), drug-induced hypertensive animal models (angiotensin-induced hypertension [55], L-nitro arginine methyl ester induced hypertension [56], etc.), metabolic hypertensive animal models (excessive alcohol intake and high fat diet induced hypertensive rats [16], high-purine diet induced hypertensive rats [57], high-glucose/fat diet induced hypertensive rats [58], etc.), and so on. SHR, hypertensive spontaneous rate of $100 \%$, were nurtured form Wistar rats by Okamoto and Aoki in 1963 [59], which is internationally recognized as the most comparable in characteristics with human essential hypertension (EH). With the development of the disease, SHR present heart [37], brain [46], kidney [43, 44], blood vessels [26], and other types of target organ damage. Classic BPV animal model, with surgery to remove sinus nerve bow (sinoaortic-denervated, SAD), was created successfully by the Krieger in 1964 [60], with the limitations of a high mortality rate and pure neurogenicity [61]. However, the BPV of SHR was positively correlated with it age. The BPV in 40-week-old SHR is higher than that in 16-week-old SHR
[62] and the BPV in 7-month-old and 5-month-old SHR is higher than that in 3-month-old SHR [63]. So, we selected the 48-week-old SHR to evaluate the protective effect of PG on $\mathrm{BPV}$ and TOD in present research. Continuously monitoring 24-hour blood pressure is the basic guarantee of evaluation BPV. Methods are mainly application with noninvasive telemetry system [64], implantable telemetry technology [17, 18], and conscious and freely moving animals dynamic blood pressure analysis system [19]. Noninvasive telemetry system is mainly used for monitoring 24-hour blood pressure of human and large animals as dogs and monkeys, with a vest and no surgery. Implantable telemetry technology, with the signal transmitter and the pressure signal device embedded in the abdominal cavity, can collect data in conscious and freely moving animals for a long-term, when the animals return to normal after surgery. Using implantable telemetry technology could reduce pain and stress of animals and reduce the number of animals by improving data accuracy and selfcontrol [65]. Conscious and freely moving animals dynamic blood pressure analysis system, with arterial catheterization and continuous heparinization to ensure the transmission of the signal, will produce a certain degree of pain and stress and could not collect data for a long-term [63]. Therefore, 


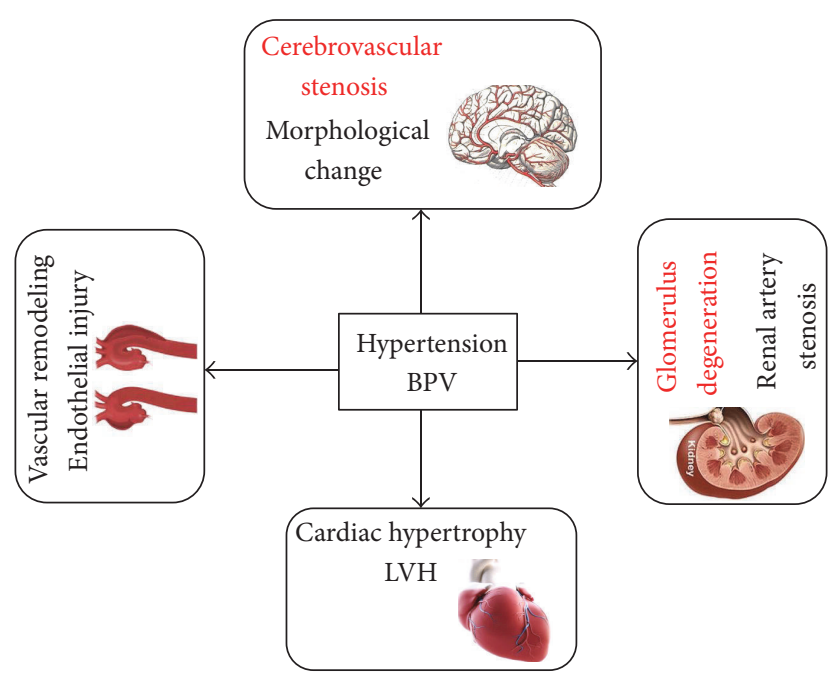

FIGURE 12: Hypertensive target organ damage (TOD) in different tissues.

to evaluate the protective effect of PG on BPV, implantable telemetry technology (Figure 2) was performed to monitor the 24-hour BPV in 3 SHR by self-control, and conscious and freely moving animals dynamic blood pressure analysis system (Figure 3 ) were performed to monitor the 24-hour BPV in 32 SHR.

In conclusion, abnormal BPV and TOD of heart, brain, kidney, and aorta were observed in SHR of this study, which is consistent with the other researcher reports. And paeoniflorin enriched extract (PG) could reduce BPV, stabilize blood pressure, and reverse the eNOS or COX-2 expression to mitigate target organ damage (TOD) in SHR. These findings provide convincing evidence that $\mathrm{PG}$, with protective effect on BPV and TOD in SHR, can be used to treat hypertension. However, the mechanisms of the increased BPV resulting in TOD were not elucidated in the present study, which may be related to chronic inflammation [66] and microcirculation [67].

\section{Competing Interests}

The authors declare that they have no conflict of interests.

\section{Authors' Contributions}

Bo Li and Zheng-Biao Yang contributed equally to the work.

\section{Acknowledgments}

The reported work was supported by China National Natural Science Foundation (81274123 to Lv Guiyuan and 81374003 to Chen Suhong), the National Science and Technology on New Drug Creation and Development Projects (2011ZX09101-002-07 to Chen Suhong and 2009ZX09502-016 to Lv Guiyuan), and the Zhe-jiang Provincial Key Laboratory Project (2012E10002 to Lv Guiyuan).

\section{References}

[1] P. Primatesta and N. R. Poulter, "Improvement in hypertension management in England: results from the Health Survey for England 2003," Journal of Hypertension, vol. 24, no. 6, pp. 11871192, 2006.

[2] P. M. Kearney, M. Whelton, K. Reynolds, P. Muntner, P. K. Whelton, and J. He, "Global burden of hypertension: analysis of worldwide data," The Lancet, vol. 365, no. 9455, pp. 217-223, 2005.

[3] L. Cai, L. Zhang, A. Liu, S. Li, and P. Wang, "Prevalence, awareness, treatment, and control of hypertension among adults in Beijing, China," Clinical \& Experimental Hypertension, vol. 19, no. 2, pp. 159-168, 2012.

[4] G. Parati, G. Pomidossi, F. Albini, D. Malaspina, and G. Mancia, "Relationship of 24-hour blood pressure mean and variability to severity of target-organ damage in hypertension," Journal of Hypertension, vol. 5, no. 1, pp. 93-98, 1987.

[5] L. Zhang, Y. Cheng, and D. Wang, "Circadian profiles of blood pressure and blood pressure variability in spontaneous hypertensive rats," Journal of Clinical Cardiology, vol. 12, no. 4, pp. 239-241, 1996.

[6] D.-F. Su and C.-Y. Miao, "Reduction of blood pressure variability: a new strategy for the treatment of hypertension," Trends in Pharmacological Sciences, vol. 26, no. 8, pp. 388-390, 2005.

[7] M.-C. Irigoyen, K. De Angelis, F. dos Santos, D. R. Dartora, B. Rodrigues, and F. M. Consolim-Colombo, "Hypertension, blood pressure variability, and target organ lesion," Current Hypertension Reports, vol. 18, no. 4, article 31, 2016.

[8] S. Xiong and Y. Wang, "Simultaneous determination of paeoniflorin from total glucosides of paeony in Sprague-Dawley rats and spontaneously hypertensive rats by high-performance liquid chromatography-tandem mass spectrometry: in vivo and in vitro studies," Biomedical Chromatography, vol. 30, no. 11, pp. 1766-1771, 2016.

[9] S. N. Jin, J. F. Wen, T. T. Wang, D. G. Kang, H. S. Lee, and K. W. Cho, "Vasodilatory effects of ethanol extract of Radix Paeoniae Rubra and its mechanism of action in the rat aorta," Journal of Ethnopharmacology, vol. 142, no. 1, pp. 188-193, 2012.

[10] G. Qian, J. Cao, C. Chen et al., "Paeoniflorin inhibits pulmonary artery smooth muscle cells proliferation via upregulating $\mathrm{A} 2 \mathrm{~B}$ adenosine receptor in rat," PLOS ONE, vol. 8, no. 7, Article ID e69141, 2013.

[11] R.-B. Guo, G.-F. Wang, A.-P. Zhao, J. Gu, X.-L. Sun, and G. Hu, "Paeoniflorin protects against ischemia-induced brain damages in rats via inhibiting MAPKs/NF- $\kappa$ B-mediated inflammatory responses," PLoS ONE, vol. 7, no. 11, Article ID e49701, 2012.

[12] Z. X. Peng, H. D. Huang, and S. G. Deng, "Effects of paeoniflorin on isolated thoracic aorta rings of rats and its possible mechanism," Chinese Journal of Experimental Traditional Medical Formulae, vol. 17, no. 7, pp. 190-193, 2011.

[13] L. Han, X. H. Zhou, W. W. Wang, and C. X. Chen, "Experimental researches of glucosides of radix paeoniae alba on reversing myocardial remodeling induced by different drugs," Journal of Liaoning University of Traditional Chinese Medicine, vol. 13, no. 2, pp. 43-48, 2011.

[14] J.-T. Cheng, C.-J. Wang, and F.-L. Hsu, "Paeoniflorin reverses guanethidine-induced hypotension via activation of central adenosine A1 receptors in wistar rats," Clinical \& Experimental Pharmacology \& Physiology, vol. 26, no. 10, pp. 815-816, 1999.

[15] C. Su-Hong, C. Qi, L. Bo, G. Jian-Li, S. Jie, and L. Gui-Yuan, "Antihypertensive effect of radix paeoniae alba in spontaneously 
hypertensive rats and excessive alcohol intake and high fat diet induced hypertensive rats," Evidence-based Complementary and Alternative Medicine, vol. 2015, Article ID 731237, 8 pages, 2015.

[16] Q. Chen, S. H. Chen, and G. Y. Lv, "Effects of herbaceous peony extract on blood pressure,blood lipid levels and hepatic function of aged apontaneously hypertensive rats," Journal of Zhejiang Chinese Medical University, vol. 37, no. 2, pp. 187-191, 2013.

[17] B. N. Van Vliet, J. McGuire, L. Chafe, A. Leonard, A. Joshi, and J.-P. Montani, "Phenotyping the level of blood pressure by telemetry in mice," Clinical \& Experimental Pharmacology \& Physiology, vol. 33, no. 11, pp. 1007-1015, 2006.

[18] E. Gillis, J. Mooney, M. Garrett, and J. Sasser, "Sildenafil treatment improves the maternal syndrome in the preeclamptic Dahl salt sensitive (s) rat," International Journal of Gynecological Cancer, vol. 20, no. 7, pp. 1197-1200, 2010.

[19] Z.-Z. Shan, S.-M. Dai, F. Fang, and D.-F. Su, "Cardiovascular and renal morphological changes in sinoaortic denervated rats," Chinese Journal of Pharmacology \& Toxicology, vol. 17, no. 2, pp. 100-105, 2003.

[20] A. J. Webb, U. Fischer, Z. Mehta, and P. M. Rothwell, "Effects of antihypertensive-drug class on interindividual variation in blood pressure and risk of stroke: a systematic review and metaanalysis," The Lancet, vol. 375, no. 9718, pp. 906-915, 2010.

[21] G. Parati, J. E. Ochoa, C. Lombardi, and G. Bilo, "Assessment and management of blood-pressure variability," Nature Reviews Cardiology, vol. 11, no. 6, pp. 143-155, 2014.

[22] X. Zhang and Q. Zhang, "Preliminary study of blood pressure variability (BPV) and target organ damage in elderly hypertension," International Journal of Cardiology, vol. 152, pp. S20-S21, 2011.

[23] S. Shibasaki, S. Hoshide, and K. Kario, "Within-home blood pressure variability on a single occasion has clinical significance," Pulse, vol. 4, no. 1, pp. 38-42, 2016.

[24] U. Fischer, A. J. S. Webb, S. C. Howard, and P. M. Rothwell, "Reporting of consistency of blood pressure control in randomized controlled trials of antihypertensive drugs: a systematic review of 1372 trial reports," Journal of Hypertension, vol. 30, no. 7, pp. 1271-1276, 2012.

[25] D.-F. Su and C.-Y. Miao, "Blood pressure variability and organ damage," Clinical and Experimental Pharmacology and Physiology, vol. 28, no. 9, pp. 709-715, 2001.

[26] K. Magyar, L. Deres, K. Eros et al., "A quinazoline-derivative compound with PARP inhibitory effect suppresses hypertension-induced vascular alterations in spontaneously hypertensive rats," Biochimica et Biophysica Acta-Molecular Basis of Disease, vol. 1842, no. 7, pp. 935-944, 2014.

[27] J.-X. Zhang, J.-R. Yang, G.-X. Chen et al., "Sesamin ameliorates arterial dysfunction in spontaneously hypertensive rats via downregulation of NADPH oxidase subunits and upregulation of eNOS expression," Acta Pharmacologica Sinica, vol. 34, no. 7, pp. 912-920, 2013.

[28] K.-T. Kang, "Endothelium-derived relaxing factors of small resistance arteries in hypertension," Toxicological Research, vol. 30, no. 3, pp. 141-148, 2014.

[29] C. Perrino, S. V. Naga Prasad, L. Mao et al., "Intermittent pressure overload triggers hypertrophy-independent cardiac dysfunction and vascular rarefaction," Journal of Clinical Investigation, vol. 116, no. 6, pp. 1547-1560, 2006.

[30] P. Martinka, J. Fielitz, A. Patzak, V. Regitz-Zagrosek, P. B. Persson, and H. M. Stauss, "Mechanisms of blood pressure variability-induced cardiac hypertrophy and dysfunction in mice with impaired baroreflex," American Journal of Physiology - Regulatory Integrative and Comparative Physiology, vol. 288, no. 3, pp. R767-R776, 2005.

[31] A. Frattola, G. Parati, C. Cuspidi, F. Albini, and G. Mancia, "Prognostic value of 24-hour blood pressure variability," Journal of Hypertension, vol. 11, no. 10, pp. 1133-1137, 1993.

[32] A. Tatasciore, M. Zimarino, R. Tommasi et al., "Increased shortterm blood pressure variability is associated with early left ventricular systolic dysfunction in newly diagnosed untreated hypertensive patients," Journal of Hypertension, vol. 31, no. 8, pp. 1653-1661, 2013.

[33] J. Ryu, R.-H. Cha, D. K. Kim et al., “The clinical association of the blood pressure variability with the target organ damage in hypertensive patients with chronic kidney disease," Journal of Korean Medical Science, vol. 29, no. 7, pp. 957-964, 2014.

[34] G. Leoncini, F. Viazzi, G. Storace, G. Deferrari, and R. Pontremoli, "Blood pressure variability and multiple organ damage in primary hypertension," Journal of Human Hypertension, vol. 27, no. 11, pp. 663-670, 2013.

[35] M. F. Hussein, A. H. Al-Mashhadani, and S. I. Essa, "The use of left ventricular myocardial stiffness index as a predictor of myocardial performance in patients with systemic hypertension," International Journal of Medical Physics, Clinical Engineering and Radiation Oncology, vol. 3, no. 3, pp. 167-175, 2014.

[36] W. B. Zhang, Q. J. Du, H. Li et al., "The therapeutic effect of Rosuvastatin on cardiac remodelling from hypertrophy to fibrosis during the end-stage hypertension in rats," Journal of Cellular \& Molecular Medicine, vol. 16, no. 9, pp. 2227-2237, 2012.

[37] L. Yan, J. D. Zhang, B. Wang et al., "Quercetin inhibits left ventricular hypertrophy in spontaneously hypertensive rats and inhibits angiotensin II-induced H9C2 cells hypertrophy by enhancing PPAR- $\gamma$ expression and suppressing AP-1 activity," PLOS ONE, vol. 8, no. 9, Article ID e72548, 2013.

[38] H. A. Baba and J. Wohlschlaeger, "Morphological and molecular changes of the myocardium after left ventricular mechanical support," Current Cardiology Reviews, vol. 4, no. 3, pp. 157-169, 2008.

[39] Y. Qian, P. Zhong, D. Liang et al., "A newly designed curcumin analog Y20 mitigates cardiac injury via anti-inflammatory and anti-oxidant actions in obese rats," PLoS ONE, vol. 10, no. 3, Article ID e0120215, 2015.

[40] T. A. Samad, K. A. Moore, A. Sapirstein et al., "Interleukin$1 \beta$-mediated induction of Cox- 2 in the CNS contributes to inflammatory pain hypersensitivity," Nature, vol. 410, no. 6827, pp. 471-475, 2001.

[41] R.-B. Guo, G.-F. Wang, A.-P. Zhao, J. Gu, X.-L. Sun, and G. Hu, "Paeoniflorin protects against ischemia-induced brain damages in rats via inhibiting MAPKs/NF- $\kappa$ B-Mediated inflammatory responses," PLoS ONE, vol. 7, no. 11, Article ID e49701, 2012.

[42] Z. Jia and J. He, "Paeoniflorin ameliorates rheumatoid arthritis in rat models through oxidative stress, inflammation and cyclooxygenase 2," Experimental and Therapeutic Medicine, vol. 11, no. 2, pp. 655-659, 2016.

[43] H. Chen, J. Yin, Y. Deng et al., "The protective effects of ginsenoside Rgl against hypertension target-organ damage in spontaneously hypertensive rats," BMC Complementary \& Alternative Medicine, vol. 12, article no. 53, 2012.

[44] D. Tian, S. Ling, G. Chen et al., "Hypertensive nephropathy treatment by heart-protecting musk pill: a study of antiinflammatory therapy for target organ damage of hypertension," International Journal of General Medicine, vol. 4, pp. 131-139, 2011. 
[45] P. Palatini, "Day-by-day blood pressure variability: cause or consequence of vascular brain injury?" Hypertension, vol. 63, no. 6, pp. 1163-1165, 2014.

[46] D. Kaiser, G. Weise, K. Möller et al., "Spontaneous white matter damage, cognitive decline and neuroinflammation in middleaged hypertensive rats: an animal model of early-stage cerebral small vessel disease," Acta neuropathologica communications, vol. 2, article 169, 2014.

[47] H. Liu, J. Wang, J. Wang, P. Wang, and Y. Xue, "Paeoniflorin attenuates $\mathrm{A} \beta 1-42$-induced inflammation and chemotaxis of microglia in vitro and inhibits NF- $\kappa$ B- and VEGF/Flt-1 signaling pathways," Brain Research, vol. 1618, pp. 149-158, 2015.

[48] H.-R. Zhang, J.-H. Peng, X.-B. Cheng, B.-Z. Shi, M.-Y. Zhang, and R.-X. Xu, "Paeoniflorin atttenuates amyloidogenesis and the inflammatory responses in a transgenic mouse model of Alzheimer's disease," Neurochemical Research, vol. 40, no. 8, pp. 1583-1592, 2015.

[49] J.-Y. Wang, H. Li, C.-M. Ma, J.-L. Wang, X.-S. Lai, and S.-F. Zhou, "MicroRNA profiling response to acupuncture therapy in spontaneously hypertensive rats," Evidence-based Complementary and Alternative Medicine, vol. 2015, Article ID 204367, 9 pages, 2015.

[50] G. Luo, X. Zhu, Z. Gao et al., "Effects of Yishen Pinggan Recipe on renal protection and NF- $\kappa$ B signaling pathway in spontaneously hypertensive rats," Evidence-Based Complementary and Alternative Medicine, vol. 2016, Article ID 6435040, 7 pages, 2016.

[51] S. Rubattu, S. Di Castro, M. Cotugno et al., "Protective effects of Brassica oleracea sprouts extract toward renal damage in highsalt-fed SHRSP: role of AMPK/PPAR $\alpha / \mathrm{UCP} 2$ axis," Journal of Hypertension, vol. 33, no. 7, pp. 1465-1479, 2015.

[52] B. Mell, V. R. Jala, A. V. Mathew et al., "Evidence for a link between gut microbiota and hypertension in the Dahl rat," Physiological Genomics, vol. 47, no. 6, pp. 187-197, 2015.

[53] E. Oliveirasales, E. Maquigussa, V. Varela et al., "Mesenchymal stem cells (MSC) improve both stenotic and contralateral kidneys in the renovascular hypertension," The FASEB Journal, vol. 29, no. 1, pp. 960-975, 2015.

[54] M. Zhou, L. Mao, Y. Wang et al., "Morphologic changes of cerebral veins in hypertensive rats: venous collagenosis is associated with hypertension," Journal of Stroke \& Cerebrovascular Diseases, vol. 24, no. 3, pp. 530-536, 2015.

[55] M. R. Bersi, C. Bellini, J. Wu, K. R. C. Montaniel, D. G. Harrison, and J. D. Humphrey, "Excessive adventitial remodeling leads to early aortic maladaptation in angiotensin-induced hypertension," Hypertension, vol. 67, no. 5, pp. 890-896, 2016.

[56] S. A. Sadek, L. A. Rashed, A. M. Bassam, and E. S. Said, "Effect of aliskiren, telmisartan and torsemide on cardiac dysfunction in 1-nitro arginine methyl ester (1-NAME) induced hypertension in rats," Journal of Advanced Research, vol. 6, no. 6, pp. 967-974, 2015.

[57] Q. Zhang, G. Y. Lv, C. Q. Xia et al., "A novel hypertensive rat model induced by long-term high-purine diet," Pharmacology \& Clinics of Chinese Materia Medica, vol. 29, no. 1, pp. 159-162, 2013.

[58] C. Q. Xia, G. Y. Lv, S. H. Chen et al., "Effect of long-term high glucose and lysophosphatidylcholine diet on blood pressure, liver and kidney function in rats," Pharmacology \& Clinics of Chinese Materia Medica, vol. 28, no. 3, pp. 122-124, 2012.

[59] K. Okamoto and K. Aoki, "Development of a strain of spontaneously hypertensive rats," Japanese circulation journal, vol. 27, no. 3, pp. 282-293, 1963.
[60] E. M. Krieger, "Neurogenic hypertension in the rat," Circulation Research, vol. 15, no. 6, pp. 511-521, 1964.

[61] J. S. del Mauro, Y. Santander, F. M. Bertera et al., "Effects of chronic carvedilol administration on blood pressure variability and target organ injury in rats with sinoaortic denervation," Revista Argentina De Cardiologia, vol. 83, no. 3, pp. 181-186, 2015.

[62] L. Hong, C. S. Xiao, L. Wang et al., "Changes of ambulatory blood pressure and morphology of resistance vessel in spontaneously hypertensive rats at different weeks of ages," Chinese Journal of Pathophysiology, vol. 27, no. 4, pp. 653-657, 2011.

[63] L. Z. Zhang, S. Z. Pan, W. M. Zhou et al., "Study on dynamic changes and gender difference of heart rate variability and blood pressure variability via implantable telemetry technology in spontaneously hypertensive rats," Chinese Journal of Comparative Medicine, vol. 25, no. 1, pp. 15-19, 2015.

[64] B. Schmelting, M. Niehoff, B. Egner, S. H. Korte, and G. F. Weinbauer, "High definition oscillometry: a novel technique for non-invasive blood pressure monitoring in the cynomolgus monkey (Macaca fascicularis)," Journal of Medical Primatology, vol. 38, no. 5, pp. 293-301, 2009.

[65] J. A. Potkay, "Long term, implantable blood pressure monitoring systems," Biomedical Microdevices, vol. 10, no. 3, pp. 379392, 2008.

[66] H. Kai, H. Kudo, N. Takayama, S. Yasuoka, Y. Aoki, and T. Imaizumi, "Molecular mechanism of aggravation of hypertensive organ damages by short-term blood pressure variability," Current Hypertension Reviews, vol. 10, no. 3, pp. 125-133, 2014.

[67] C.-H. Chen and M.-H. Shyr, "Blockade of spinal nitric oxide synthase on blood pressure variability and hepatic microcirculation," Acta Anaesthesiologica Taiwanica, vol. 43, no. 2, pp. 67$72,2005$. 


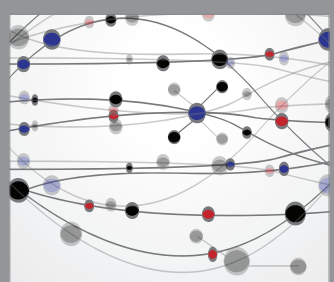

The Scientific World Journal
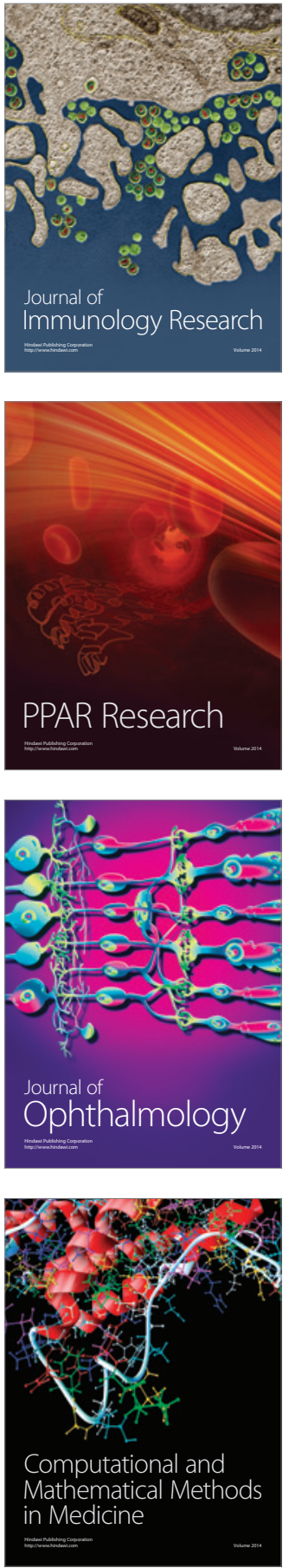

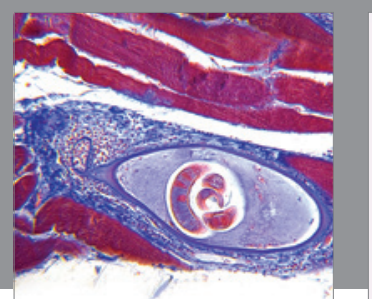

Gastroenterology Research and Practice
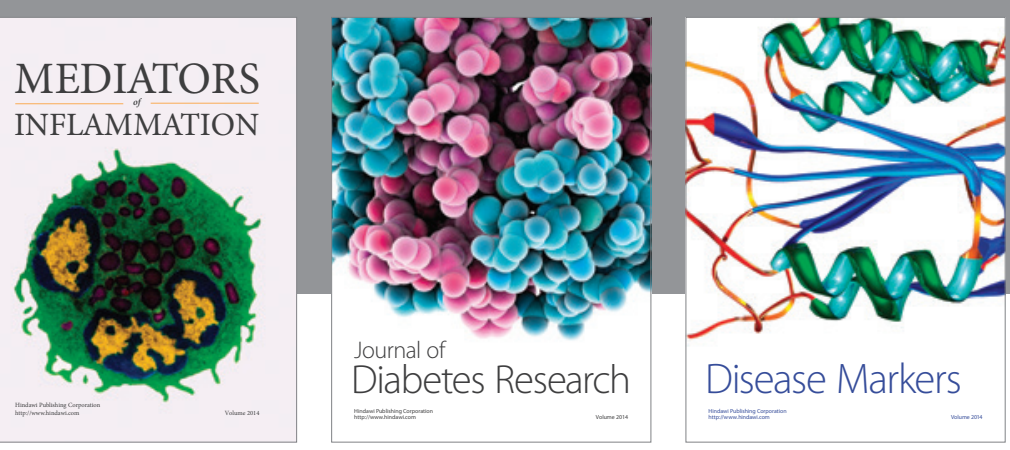

Disease Markers

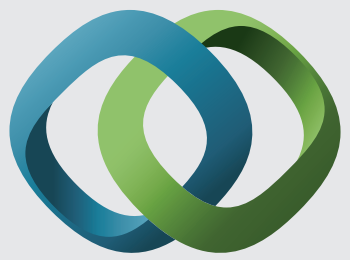

\section{Hindawi}

Submit your manuscripts at

https://www.hindawi.com
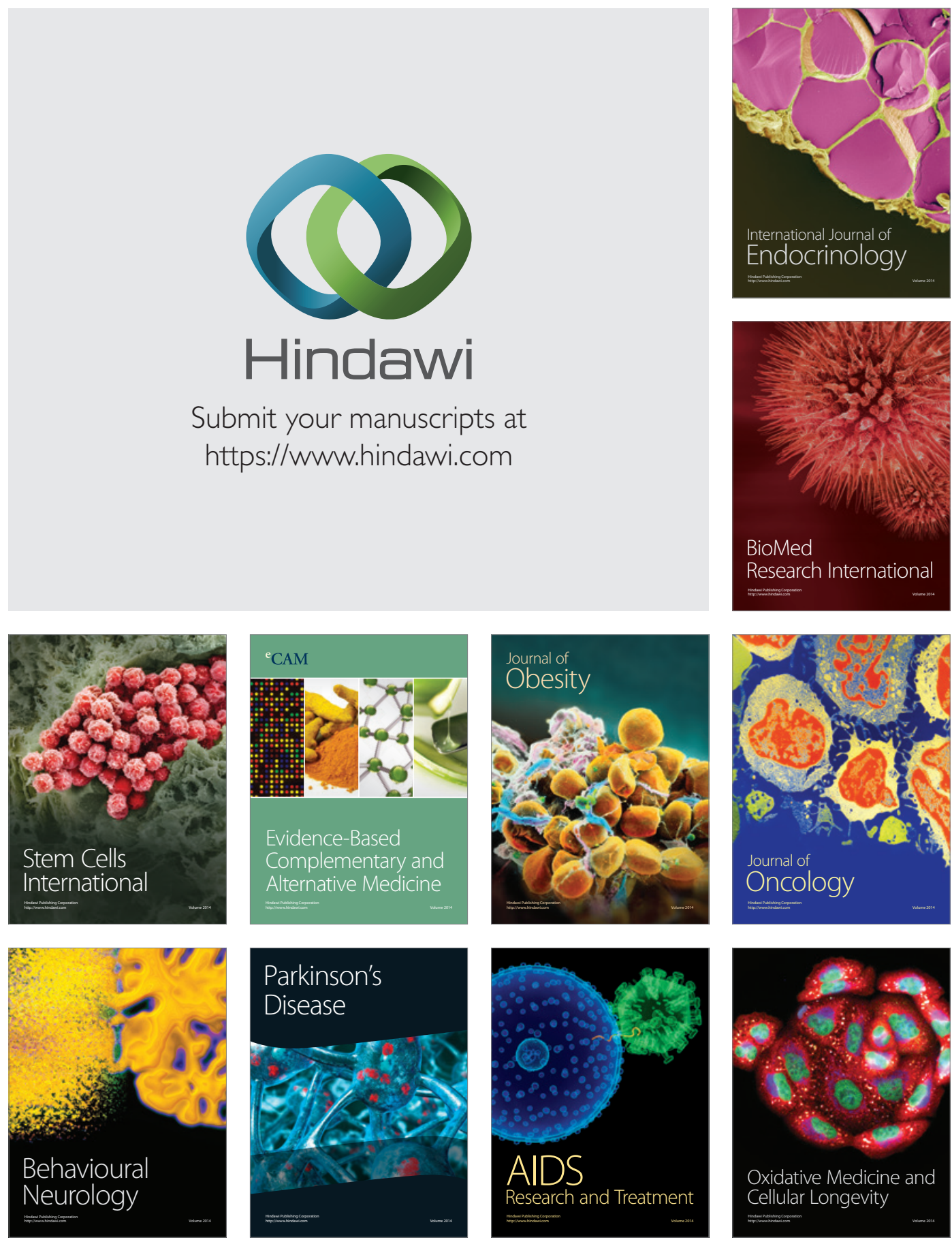